\title{
Approximate solutions to the initial value problem for some compressible flows
}

\author{
M. Colombeau
}

\begin{abstract}
For the natural initial conditions $L^{1}$ in the density field (more generally a positive bounded Radon measure) and $L^{\infty}$ in the velocity field, we obtain global approximate solutions to the Cauchy problem for the 3-D systems of isothermal and isentropic gases and the 2-D shallow water equations. We obtain a sequence of functions which are differentiable in time and continuous in space and tend to satisfy the equations in the sense of distributions in the space variables and in the strong sense in the time variable. The method of construction relies on the study of a specific family of two ODEs in a classical Banach space (one for the continuity equation and one for the Euler equation). Standard convergent numerical methods for the solution of these ODEs can be used to provide concrete approximate solutions. It has been checked in numerous cases in which the solutions of systems of fluid dynamics are known that our construction always gives back the known solutions. It is also proved that it gives the classical analytic solutions in the domain of application of the Cauchy-Kovalevskaya theorem.
\end{abstract}

Mathematics Subject Classification. 35D30 - 35Q31 - 35Q35.

Keywords. Partial differential equations · Numerical analysis · Functional analysis · Weak asymptotic solutions · Fluid dynamics.

\section{Introduction}

We construct sequences of approximate solutions with arbitrary accuracy for the 1-D, 2-D and 3-D compressible isothermal gases and isentropic gases in the presence of shocks and void regions; this applies also to the shallow water equations with same proof. The need for the search of mathematical solutions to the initial value problem for compressible fluids in several dimensions when shocks show up has been recently pointed out by Lax and Serre [25,31]; our results provide sequences of approximate solutions with full mathematical proofs that tend to satisfy the equations. We have been motivated by the fact our approximate solutions are weak asymptotic solutions as considered by Albeverio et al. [1-3], Danilov et al. [15-18], Shelkovich et al. [30,32,33], as an extension of the Maslov-Whitham asymptotic analysis. Exact or approximate solutions with full proofs in the case of spherical symmetry had already been obtained in Joseph et al. [4,22] and Kunzinger et al. [23,24] for some of these systems or related systems. The proofs are given in 1-D to permit a better understanding thanks to simplification in notation. Then we check in detail that the proofs extend in a straightforward way in multi-D. We give four numerical tests in 1-D and two numerical tests in 2-D: creation of a void region in the 2-D isentropic case [19] and the cylindrical dam break problem of Toro [35].

The starting point of our attempt [7-9] was the search of a numerical scheme permitting mathematical proofs of partial results aiming to connect the step functions produced by the scheme and the system one tries to solve: In the absence of mathematically well-defined solutions that could permit to investigate convergence to these solutions, it is natural to try to prove that the step functions resulting from the scheme tend to satisfy the equations, which makes sense in the absence of properly defined mathematical solutions. Sequences of functions that tend to satisfy the equations have been already considered as a

This research has been supported by FAPESP, processo 2012/15780-9. 
tool in itself by various authors following Maslov asymptotic analysis [1-3,15-18,30,32,33], and also, they could be a provisional substitute of solutions, since one has to cope with an absence of known usual weak solutions in 2-D and 3-D in the presence of shocks. The proof that these sequences tend to satisfy the equations permits to explain results observed from computing in $[9,25,26]$. In order to obtain full proofs, we replaced the original scheme [7-9] by ODEs in Banach spaces whose solutions provide the sequence of "approximate solutions," at the same time as one retains the possibility of computing the solutions of these ODEs to check that one obtains the correct results.

From a physical viewpoint, the equations of fluid dynamics are marked with some imprecision since they do not take into account some minor effects and the molecular structure of matter. It is natural to expect that these equations and their imprecision should be stated in the sense of distributions in the space variables. Sequences $[(x, y, z, t) \longmapsto U(x, y, z, t, \epsilon)]_{\epsilon}$ of approximate solutions in the sense of distributions enter into this imprecision for $\epsilon>0$ small enough. Therefore, these sequences appear to provide a natural representation of physical solutions. Of course one has checked that the sequences of approximate solutions we construct always give the correct solution in all the numerous tests that were performed.

In the case of scalar conservation laws, the sequences of approximate solutions constructed by the method in this paper tend to the Krushkov entropy solution when $\epsilon \rightarrow 0$ [13]. For the equations of fluid dynamics considered here, in absence of a known similar uniqueness and well-posedness result, except in the Cauchy-Kovalevskaya case which is far too restrictive, one has to content with numerical confirmations from faithful computer calculations.

These sequences of approximate solutions have been introduced under the name of weak asymptotic solutions by Danilov et al. [15], as an extension of Maslov asymptotic analysis, and they have proved to be an efficient mathematical tool to study creation and superposition of singular solutions to various nonlinear PDEs, in particular $\delta$-waves: $[1-3,15-18,30,32,33]$. A weak asymptotic solution for the system

$$
\partial_{t} U_{i}+\partial_{x} f_{i}\left(U_{1}, \ldots, U_{p}\right)+\partial_{y} g_{i}\left(U_{1}, \ldots, U_{p}\right)=0,1 \leq i \leq p, U_{i}: \mathbb{R}^{2} \times \mathbb{R}^{+} \mapsto \mathbb{R},
$$

is a sequence $\left(U_{1, \epsilon}, \ldots, U_{p, \epsilon}\right)_{\epsilon}$ of functions such that $\forall i \in\{1, \ldots, p\}, \forall \psi \in \mathcal{C}_{c}^{\infty}\left(\mathbb{R}^{2}\right) \forall t$

$$
\int_{\mathbb{R}^{2}}\left[\partial_{t} U_{i, \epsilon} \psi-f_{i}\left(U_{1, \epsilon}, \ldots, U_{p, \epsilon}\right) \partial_{x} \psi-g_{i}\left(U_{1, \epsilon}, \ldots, U_{p, \epsilon}\right) \partial_{y} \psi\right] \mathrm{d} x \mathrm{~d} y \rightarrow 0
$$

when $\epsilon \rightarrow 0$ if we consider the strong derivative in $t$ and weak derivatives in $x, y$ as this will be the case in this paper. In short, the sequence $\left(U_{1, \epsilon}, \ldots, U_{p, \epsilon}\right)_{\epsilon}$ tends to satisfy the equations when $\epsilon \rightarrow 0$. Of course the $U_{i, \epsilon}^{\prime} s$ are also chosen so as to satisfy the initial condition stated in a natural sense.

The approximate solutions that we construct satisfy uniform $L^{1}$ bounds in density (consequence of mass conservation) but, in the presence of pressure, one does not obtain a bounded velocity; this should not be too much unexpected: Indeed, various instances are known in which the ideal inviscid equations give infinite velocity [21] sections 6.6.2, 7.4.3, [14] section 14.9.1. The concept of sequences of approximate solutions in this paper permits infinite velocity at the limit $\epsilon \rightarrow 0$.

We construct a weak asymptotic solution for the 3-D systems of isothermal and isentropic gases that we state in $2-\mathrm{D}$ for convenience (immediate extension to $3-\mathrm{D}$ ) in the form

$$
\begin{aligned}
& \partial_{t} \rho+\partial_{x}(\rho u)+\partial_{y}(\rho v)=0, \\
& \partial_{t}(\rho u)+\partial_{x}\left(\rho u^{2}\right)+\partial_{y}(\rho u v)+\partial_{x} p=0, \\
& \partial_{t}(\rho v)+\partial_{x}(\rho u v)+\partial_{y}\left(\rho v^{2}\right)+\partial_{y} p=0, \\
& p=K \rho, \quad \text { (isothermal gases }), \\
& p=K \rho^{\gamma}, \quad 1<\gamma \leq 2, \quad \text { (isentropic gases). }
\end{aligned}
$$


The notation : $\rho=\rho(x, y, t)$ is the gas density, $(u, v)=(u(x, y, t), v(x, y, t))$ is the velocity vector in the $x, y$ directions, respectively, $p=p(x, y, t)$ is the pressure, and $K$ is a constant. The problem is studied on the $n$-dimensional torus $\mathbb{T}^{n}=\mathbb{R}^{n} /(2 \pi \mathbb{Z})^{n}, n=1,2,3$.

Then, since the equations are similar, we construct weak asymptotic solutions for the 2-D shallow water equations stated in the form

$$
\begin{aligned}
\partial_{t} h+\partial_{x}(h u)+\partial_{y}(h v) & =0, \\
\partial_{t}(h u)+\partial_{x}\left(h u^{2}\right)+\partial_{y}(h u v)+g h \partial_{x}(h+a) & =0, \\
\partial_{t}(h v)+\partial_{x}(h u v)+\partial_{y}\left(h v^{2}\right)+g h \partial_{y}(h+a) & =0,
\end{aligned}
$$

where $h=h(x, y, t)$ is the water elevation, $(u, v)=(u(x, y, t), v(x, y, t))$ is the velocity vector in the $x, y$ directions, respectively, $a=a(x, y)$ is the bottom elevation assumed to be of class $\mathcal{C}^{2}$ and $g=9.8$. The problem is studied on the $n$-dimensional torus $\mathbb{T}^{n}, n=1,2$.

The method of proof relies on the statement and study of a system of ODEs (one for the continuity equation, one for the Euler equation) in the Banach space $\mathcal{C}\left(\mathbb{T}^{n}\right), n=1,2,3$, of continuous functions on the $n$-dimensional torus. We expose in detail the proof in 1-D for simplification in notation, and then, we check that its extension to multi-dimension is only a matter of notation. One uses the theory of ODEs in the Lipschitz case, and a priori estimates needed to prove existence of a global in positive time solution. It has been inspired by the method introduced in [11] in the pressureless case and in [10] to put in evidence continuations of the analytic solutions after the time of the analytic blow up.

Numerical calculations of these approximate solutions can be done easily by solving the above ODEs in Banach space by classical convergent numerical methods for ODEs such as the explicit Euler order one method or the Runge-Kutta RK4 method. This has given the known solutions in all 1-D and 2-D tests, which could have been expected since the method in this paper is issued from the numerical scheme in [7-9]. For this scheme a large amount of numerical verifications for initial conditions in which the solution is known has been done on classical and demanding benchmarks of scientific computing (Sod [34], Woodward-Colella [38], Toro [35,36], Lax [25,26], Bouchut et al. [5], LeVeque [27], Chertock et al. [6], Evje and Flatten [20]).

\section{Approximate solutions to the system of isothermal gases and numerical confirmations}

We state the classical system of isothermal gases in 1-D in the form:

$$
\begin{aligned}
& \partial_{t} \rho+\partial_{x}(\rho u)=0, \\
& \partial_{t}(\rho u)+\partial_{x}\left(\rho u^{2}\right)+\rho \partial_{x} \Phi=0, \\
& \Phi=K \ln (\rho), K=\mathrm{const} \geq 0 .
\end{aligned}
$$

Therefore, the last term in the Euler equation (11) is in the familiar form $\rho \frac{\partial}{\partial x} \Phi=\frac{\partial}{\partial x} p$, with $p=K \rho$. Here $\Phi$ is the density of the body force caused by the pressure: $\rho \vec{\nabla} \Phi=\vec{\nabla} p$. Formulation (12) excludes void regions. This apparent defect will be repaired by the term $\rho \vec{\nabla} \Phi$ in the Euler equation: It will be proved theoretically and checked numerically that the construction gives a weak asymptotic solution even in the presence of void regions. We use the notation

$$
u^{+}=\max (u, 0), \quad u^{-}=\max (-u, 0),
$$

so that

$$
u=u^{+}-u^{-}, \quad|u|=u^{+}+u^{-} .
$$

To simplify the notation, we first study the system in 1-D. The 2-D extensions of formulas $(15,16)$ below and the verification that the 1-D proof extends to multi-D in a direct way are given at the end of the proof of Theorem 1 . 
In $1-D$ we state the method as the system of $\operatorname{ODEs}(15,16)$ complemented by the formulas $(17,18)$ :

$$
\frac{\mathrm{d}}{\mathrm{d} t} \rho(x, t, \epsilon)=\frac{1}{\epsilon}\left[\left(\rho u^{+}\right)(x-\epsilon, t, \epsilon)-(\rho|u|)(x, t, \epsilon)+\left(\rho u^{-}\right)(x+\epsilon, t, \epsilon)\right]+\epsilon^{\beta},
$$

for some $\beta>0$ to be made precise later,

$$
\begin{aligned}
\frac{\mathrm{d}}{\mathrm{d} t}(\rho u)(x, t, \epsilon) & =\frac{1}{\epsilon}\left[\left(\rho u u^{+}\right)(x-\epsilon, t, \epsilon)-(\rho u|u|)(x, t, \epsilon)+\left(\rho u u^{-}\right)(x+\epsilon, t, \epsilon)\right]-\rho(x, t, \epsilon) \partial_{x} \Phi(x, t, \epsilon), \\
u(x, t, \epsilon) & =\frac{(\rho u)(x, t, \epsilon)}{\rho(x, t, \epsilon)},
\end{aligned}
$$

for which we will prove that $\rho(x, t, \epsilon)>0$, thus permitting division. This will follow from the proof by a priori inequalities exposed below, more precisely from the left-hand side of formula (26) which is proved to hold for all $t \in\left[0,+\infty[)\right.$. The term $\epsilon^{\beta}$ in (15) is needed because of the specific form of the state law of isothermal gases and is not needed for the isentropic gases and shallow water equations considered in subsequent sections.

We approximate the state law (12) in the form

$$
\Phi(x, t, \epsilon)=K\left[\ln \left(\rho(., t, \epsilon)+\epsilon^{N}\right) * \phi_{\epsilon^{\alpha}}\right](x), \quad N>0,
$$

where $K$ is the coefficient and in which the term $\epsilon^{N}$ has been introduced to permit void regions. As explained below, we introduce in (18) a convolution as some kind of averaging. To this end, we consider an auxiliary function $\phi \in \mathcal{C}_{c}^{\infty}(\mathbb{R}), \phi \geq 0, \phi(-x)=\phi(x) \forall x$ and $\int \phi(x) \mathrm{d} x=1$. If $\mu>0$ we set $\phi_{\mu}(x)=\frac{1}{\mu} \phi\left(\frac{x}{\mu}\right)$, so that the family $\left\{\phi_{\mu}\right\}_{\mu}$ tends in the sense of distributions to the Dirac measure when $\mu \rightarrow 0^{+}$. We use a real number $\alpha, 0<\alpha<1$, to be made more precise later. The convolution in (18) is justified by the fact (12) is a state law and, as such, is physically valid only in space regions larger than those in which the basic conservation laws $(10,11)$ are valid. An explanation is needed to justify the convolution in (18). The formulas $(15,16)$ state that the conservation laws of mass and momentum are valid in the cells of length $\epsilon$. These conservation laws are known to hold at a very small scale close to the molecular scale; maybe $\epsilon$ can be of the order of magnitude of $10^{-8} \mathrm{~m}$. On the other hand, the state law $p=K \rho$ is valid only at the order of magnitude of the measurement apparatus and in absence of strong irregularities such as shock waves, or a slight extrapolation at smaller order, maybe at a magnitude not smaller than $10^{-3} \mathrm{~m}$ represented in (18) by $\epsilon^{\alpha}$ in the convolution. This is the theoretical reason for which this convolution and $\epsilon^{\alpha}, 0<\alpha<1$, are introduced. Somewhat surprisingly, this modelization from physics permits on the one hand the mathematical proofs and, on the another hand, one observes in all numerical tests the need of a convolution done usually numerically by an averaging on three cells (see comments of Fig. 1), which needs to be slowly strengthened to involve more cells when $\epsilon$ becomes very small: The number of cells involved in the convolution (18) is proportional to $\frac{\epsilon^{\alpha}}{\epsilon}$.

We will prove that when the initial conditions $\rho_{0} \in L^{1}(\mathbb{T}), u^{0} \in L^{\infty}(\mathbb{T}), \rho_{0} \geq 0$ are approximated by a family $\left(\rho_{0}^{\epsilon}, u_{0}^{\epsilon}\right)$ of continuous regularizations defined on $\mathbb{T}$, such that $\rho_{0}^{\epsilon}(x)>0 \forall x,\left\|\rho_{0}-\rho_{0}^{\epsilon}\right\|_{L^{1}(\mathbb{T})} \rightarrow$ $0,\left|\left\|u_{0}\right\|_{\infty}-\left\|u_{0}^{\epsilon}\right\|_{\infty}\right| \rightarrow 0$ when $\epsilon \rightarrow 0$, then

Theorem 1. The solution of the system of ODEs (15-18), with $\alpha<\frac{1}{6}, 3 \alpha+\beta<N-1$, with initial conditions $\left(\rho_{0}^{\epsilon}, u_{0}^{\epsilon}\right)$, provides a weak asymptotic solution (1) for the 1-D isothermal gas equations, which is global in time $t \in\left[0,+\infty\left[\right.\right.$ and in space $x \in \mathbb{T}$. When $\alpha<\frac{1}{4+2 n}$ and $(2+n) \alpha+\beta<N-1$, the result holds as well in $n-D, n=2,3$ for a direct extension of $(15,18)$.

Remark 1. From the lack of uniqueness of a limit when $\epsilon \rightarrow 0$ of weak asymptotic solutions (an aspect of the well-known nonuniqueness of weak solution concepts), the most interesting point is not the existence of a weak asymptotic solution but that our particular construction gives, from a rigorous mathematical proof, a weak asymptotic solution adapted to numerical calculations, which provides a bridge between theory and numerics. 

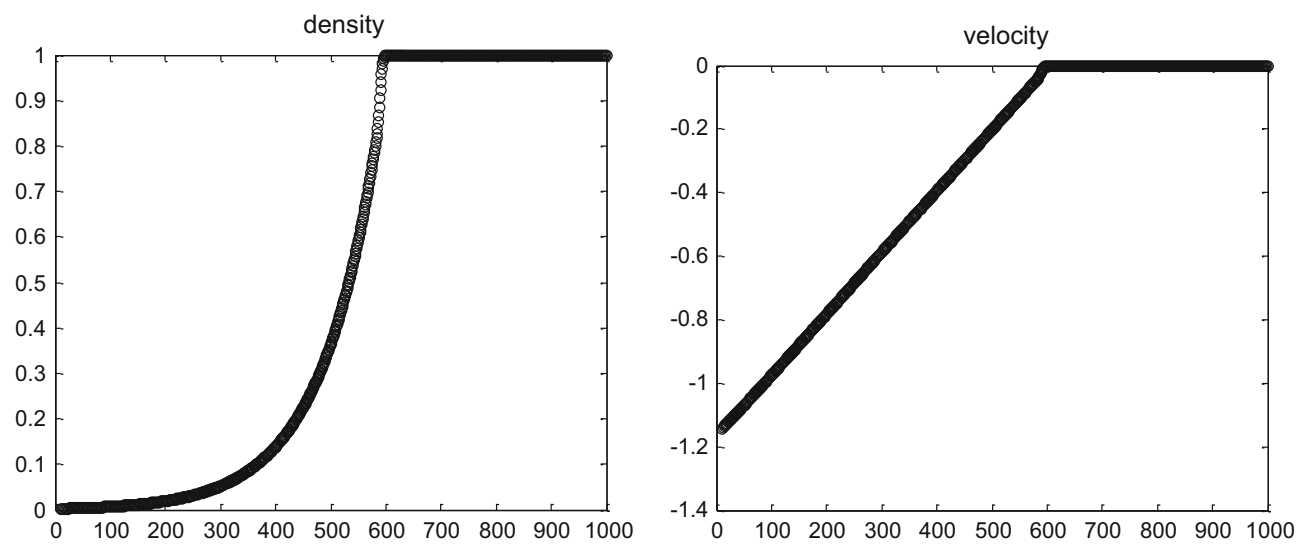

FIG. 1. A demanding test on the system of isothermal gases with a void region; left density, right velocity

Since the proof is long and occupies the end of this section, we first explain the structure of the proof before the detailed proof that follows. The proof is based on a priori inequalities obtained for the ODEs (15-18) due to their specific form issued from physics.

1) Initial conditions First, we replace the initial conditions $\rho_{0}$ and $u_{0}$ by continuous regularizations $\rho_{0}^{\epsilon}$ and $u_{0}^{\epsilon}$ that tend, respectively, to $\rho_{0}$ and $u_{0}$ in $L^{1}(\mathbb{T})$. In the presence of a void region $\mathcal{R}$ in the initial conditions, we decide $\rho_{0}^{\epsilon}(x)>0 \forall \epsilon \forall x \in \mathcal{R}$, and $\rho_{0}^{\epsilon}(x) \rightarrow 0$ when $\epsilon \rightarrow 0 \forall x \in \mathcal{R}$. The void region is taken into account at the limit $\epsilon \rightarrow 0$. Therefore by continuity in time of the local solution (ODE in the Lipschitz case for each fixed value of $\epsilon$, without any uniformness in $\epsilon$ ), one will have (20) for $m=m(\epsilon)$ on some small time interval $\delta(\epsilon)>0$.

2) A priori estimates for fixed $\epsilon$ Using $m=m(\epsilon), M=M(\epsilon)$ from $(20,21)$ as auxiliary ingredients in the reasoning, one proves, as long as a solution of the ODEs $(15-18)$ exists, the estimates $(24,26)$ in Proposition 1 , in which the values $m$ and $M$ have completely disappeared.

3) The ODEs for fixed $\epsilon$ One notices that the Lipschitz coefficients of the functions $F, G(35)$ involved in the ODEs (always for fixed $\epsilon$ ) are uniformly bounded on any region of the Banach space $(\mathcal{C}(\mathbb{T}))^{2}$ for which there exist $\alpha>0$ (small) and $A>0$ (large) such that for all $x \in \mathbb{T}$ one has

$$
\rho(x) \geq \alpha \quad \text { and } \quad \rho(x) \leq A \text { and }|u(x)| \leq A .
$$

This remark implies (similarly to the case of Lipschitz ODEs in finite dimensional spaces) that the solution of the ODEs ceases to exist at a finite time $T$ only when there is a sequence $\left(t_{n}\right), t_{n}<T$ on which one at least of these three conditions is not satisfied uniformly in $n$ when $n \rightarrow \infty$.

4) Proof of global solution for fixed $\epsilon$ Assume the solution ceases to exist at a time $T<\infty$. Apply Proposition 1 in $[0, T$ [ instead of $[0, \delta(\epsilon)[$ : The a priori estimates in Proposition 1 imply that one has bounds $(24,26)$ for $t=t_{n}$ which can be stated same for all $n$ since a fortiori they apply replacing $t_{n}$ by $T$. This proves that the above three conditions are satisfied uniformly in $n$ on the left of $T$. Therefore, the solution extends on the right of $T$, which contradicts the assumption. Therefore, the solution exists globally in positive time. In particular from (26) applied on any interval $[0, t[$ and for any $\epsilon$, one has $\rho(x, t, \epsilon)>0 \forall x, t, \epsilon$.

5) Weak asymptotic solution when $\epsilon \rightarrow 0$ One proves from bounds in Proposition 1 that the solutions of the ODEs provide weak asymptotic solutions.

6) Multi-D case One checks that the 1-D proof above holds in multi-D without any other modification than notation.

Void regions One should clarify the concept of void region used in the paper. A void region $\mathcal{R}$ at time $t \geq 0$ is a region for which $\rho(x, t, \epsilon) \rightarrow 0$ when $\epsilon \rightarrow 0$ for all $x \in \mathcal{R}$. In this way, one accepts the presence of void regions (both in initial condition and at time $t$ in the solution) as well as in all proofs one works 
with $\rho(x, t, \epsilon)>0$ for each $\epsilon$. There is no harm since in physics as well as in numerical practice (in [19] a void region is defined by $\rho<10^{-14}$ ), it seems that a void region has the status of an idealization at a limit such as $\epsilon \rightarrow 0$, rather than a basic status in itself.

Now we start the detailed proof, first in the 1-D case.

Proof of Theorem 1 in the 1-D case. We first establish a priori inequalities (Proposition 1) to prove existence of a global solution to (15-18). For fixed $\epsilon>0$ and for some $\delta(\epsilon)>0$, we assume existence of a solution

$$
\begin{aligned}
{[0, \delta(\epsilon)[} & \longmapsto(\mathcal{C}(\mathbb{T}))^{2} \\
t & \longmapsto[x \mapsto(\rho(x, t, \epsilon),(\rho u)(x, t, \epsilon))]
\end{aligned}
$$

continuously differentiable on $[0, \delta(\epsilon)[$ (with a right- hand side derivative at $t=0$ ) having the following properties for each fixed $\epsilon: \forall \delta^{\prime}<\delta(\epsilon)$

$$
\begin{aligned}
& \exists m=m(\epsilon)>0 / \rho(x, t, \epsilon) \geq m \forall x \in \mathbb{T} \forall t \in\left[0, \delta^{\prime}\right], \\
& \exists M=M(\epsilon)>0 /\|u(., t, \epsilon)\|_{\infty} \leq M, \quad\|\rho(., t, \epsilon)\|_{\infty} \leq M \forall t \in\left[0, \delta^{\prime}\right] .
\end{aligned}
$$

Note that $m$ and $M$ depend on $\epsilon$ and that from the Lipschitz property of the ODEs (15-18) proved below for each $\epsilon$ there exists such a local solution provided the initial conditions $\rho_{0}^{\epsilon}(x)=\rho(x, 0, \epsilon), u_{0}^{\epsilon}(x)=$ $u(x, 0, \epsilon)$ satisfy bounds like $(20,21)$ for a smaller value of $M$ and a larger value of $m$ at time $t=0$. One will prove that the solution is global in positive time and obtain estimates on this solution. Note that $m=m(\epsilon)$ tends to 0 in the case of the presence of a void region in the initial condition; this does not cause trouble because the proof is done for fixed $\epsilon$. The quantities $m$ and $M$ are used in the reasoning to obtain a priori inequalities, and they disappear in the results (22-26).

Proposition 1. (a priori inequalities).

As soon as $(19,20,21)$ hold for some $\delta(\epsilon)>0$, one has

$$
\text { - } \forall t \in\left[0, \delta(\epsilon)\left[\int_{-\pi}^{\pi} \rho(x, t, \epsilon) \mathrm{d} x=\int_{-\pi}^{\pi} \rho_{0}^{\epsilon}(x) \mathrm{d} x+2 \pi \epsilon^{\beta} t,\right.\right.
$$

and $\exists C>0$, depending only on $\left\|\rho_{0}\right\|_{L^{1}(\mathbb{T})}$ and $\delta(\epsilon)$, not on $m, M$ and $\epsilon>0$ small, such that

$$
\begin{aligned}
& \bullet \forall t \in\left[0, \delta(\epsilon)\left[\left\|\partial_{x} \Phi(., t, \epsilon)\right\|_{\infty} \leq \frac{C}{\epsilon^{3 \alpha}}\right.\right. \\
& \bullet \forall t \in\left[0, \delta(\epsilon)\left[\|u(., t, \epsilon)\|_{\infty} \leq\left\|u_{0}^{\epsilon}\right\|_{\infty}+\frac{C}{\epsilon^{3 \alpha}} t .\right.\right.
\end{aligned}
$$

Set

$$
k(\epsilon)=\left\|u_{0}^{\epsilon}\right\|_{\infty}+\frac{C \delta(\epsilon)}{\epsilon^{3 \alpha}} .
$$

Then

$$
\bullet \forall t \in\left[0, \delta(\epsilon)\left[, \forall x \in \mathbb{R}, \rho_{0}^{\epsilon}(x) \exp \left(\frac{-k(\epsilon) t}{\epsilon}\right) \leq \rho(x, t, \epsilon) \leq 2\left\|\rho_{0}^{\epsilon}\right\|_{\infty} \exp \left(\frac{2 k(\epsilon) t}{\epsilon}\right) .\right.\right.
$$

Proof of Proposition 1. From $(14,15)$

$$
\begin{aligned}
\frac{\mathrm{d}}{\mathrm{d} t} \int_{-\pi}^{+\pi} \rho(x, t, \epsilon) \mathrm{d} x= & \frac{1}{\epsilon}\left[\int_{-\pi}^{+\pi}\left(\rho u^{+}\right)(x-\epsilon, t, \epsilon) \mathrm{d} x-\int_{-\pi}^{+\pi}\left(\rho u^{+}\right)(x, t, \epsilon) \mathrm{d} x-\int_{-\pi}^{+\pi}\left(\rho u^{-}\right)(x, t, \epsilon) \mathrm{d} x\right. \\
& \left.+\int_{-\pi}^{+\pi}\left(\rho u^{-}\right)(x+\epsilon, t, \epsilon) \mathrm{d} x\right]+2 \pi \epsilon^{\beta}=0+2 \pi \epsilon^{\beta}
\end{aligned}
$$

by periodicity of $\rho$ and $u$. 
Inequality (23) is proved as follows: (18) implies

$$
\partial_{x} \Phi(x, t, \epsilon)=K \int_{\mathbb{R}} \ln \left[\rho(x-y, t, \epsilon)+\epsilon^{N}\right] \frac{1}{\epsilon^{2 \alpha}} \phi^{\prime}\left(\frac{y}{\epsilon^{\alpha}}\right) \mathrm{d} y .
$$

Using (22) when $\rho(x-y, t, \epsilon) \geq 1$ (then $\left.0<\ln \left(\rho+\epsilon^{N}\right)<\rho \in L_{l o c}^{1}(\mathbb{R})\right)$, and, using that the above $\ln$ is bounded in absolute value by const $\ln \left(\frac{1}{\epsilon^{N}}\right)$ when $\rho(x-y, t, \epsilon)<1$, one obtains

$$
\left|\partial_{x} \Phi(x, t, \epsilon)\right| \leq \text { const } N \ln \left(\frac{1}{\epsilon}\right) \frac{1}{\epsilon^{2 \alpha}}\left\|\phi^{\prime}\right\|_{\infty} \leq C \frac{1}{\epsilon^{3 \alpha}} .
$$

Notice that from (22) $C$ depends only on $\left\|\rho_{0}\right\|_{L^{1}(\mathbb{T})}$ from the initial condition (point (1) in the above description of the structure of the proof) and on $\delta(\epsilon)$ from the last term in (22).

Now let us prove inequality (24). The proof is done for $t$ ranging in $\left[0, \delta^{\prime}\right], \delta^{\prime}<\delta(\epsilon)$ to benefit from some uniformness in $t$. We will obtain (24) with the same constant $C$ independent of $\delta^{\prime}$. From (15) and the assumption that the solution of the ODE is of class $\mathcal{C}^{1}$ on $[0, \delta(\epsilon)[$, valued in the Banach space $\mathcal{C}(\mathbb{T})$, one obtains for fixed $\epsilon>0$ (the proof is done for fixed $\epsilon$ : Values such as $\mathrm{d} t$ below do depend on $\epsilon$ and disappear in the final result) and for $\mathrm{d} t=\mathrm{d} t(\epsilon)>0$ small enough that

$$
\begin{aligned}
& \rho(x, t+\mathrm{d} t, \epsilon) \\
& \quad=\rho(x, t, \epsilon)+\frac{\mathrm{d} t}{\epsilon}\left[\left(\rho u^{+}\right)(x-\epsilon, t, \epsilon)-(\rho|u|)(x, t, \epsilon)+\left(\rho u^{-}\right)(x+\epsilon, t, \epsilon)\right]+\mathrm{d} t r(x, t, \epsilon)(\mathrm{d} t)+\epsilon^{\beta} \mathrm{d} t \\
& \quad=\frac{\mathrm{d} t}{\epsilon}\left(\rho u^{+}\right)(x-\epsilon, t, \epsilon)+\left(1-\frac{\mathrm{d} t}{\epsilon}|u|(x, t, \epsilon)\right) \rho(x, t, \epsilon)+\frac{\mathrm{d} t}{\epsilon}\left(\rho u^{-}\right)(x+\epsilon, t, \epsilon)+\mathrm{d} t r(x, t, \epsilon)(\mathrm{d} t)+\epsilon^{\beta} \mathrm{d} t .
\end{aligned}
$$

Formula (27) is the classical definition of the derivative of a map of the real variable $t$ valued in the Banach space $\mathcal{C}(\mathbb{T}), \mathrm{d} t$ is a (small) time increment, and $\mathrm{d} t r(x, t, \epsilon)(\mathrm{d} t)$ is the remainder when the variables $x \in \mathbb{T}$ and the parameter $\epsilon$ are noted explicitly. One obtains that $\|r(., t, \epsilon)(\mathrm{d} t)\|_{\infty} \rightarrow 0$ when $\mathrm{d} t \rightarrow 0$ uniformly for $t$ in a compact set of $[0, \delta(\epsilon)[:$ This follows from the mean value theorem under the form $\left\|f(t+\mathrm{d} t)-f(t)-f^{\prime}(t) \mathrm{d} t\right\| \leq \sup _{0<\theta<1}\left\|f^{\prime}(t+\theta \mathrm{d} t)-f^{\prime}(t)\right\||\mathrm{d} t|$. Notice that there is no uniformness in $\epsilon$. For $\mathrm{d} t>0$ small enough (depending on $\epsilon$ ) and $t \in\left[0, \delta^{\prime}\right]$ the single term $\left(1-\frac{\mathrm{d} t}{\epsilon}|u(x, t, \epsilon)|\right) \rho(x, t, \epsilon)$ dominates the term $\mathrm{d} t r(x, t, \epsilon)(\mathrm{d} t)$ from $(20,21)$ : Choose $\mathrm{d} t>0$ small enough so that the first term is $\geq \frac{1}{2} m$ and notice that the second term tends to 0 when $\mathrm{d} t \rightarrow 0$. Therefore since $\rho u^{ \pm} \geq 0$ one can invert $(27)$ :

$$
\begin{aligned}
\frac{1}{\rho(x, t+\mathrm{d} t, \epsilon)}= & {\left[\frac{\mathrm{d} t}{\epsilon}\left(\rho u^{+}\right)(x-\epsilon, t, \epsilon)+\left[1-\frac{\mathrm{d} t}{\epsilon}|u|(x, t, \epsilon)\right] \rho(x, t, \epsilon)\right.} \\
& \left.+\frac{\mathrm{d} t}{\epsilon}\left(\rho u^{-}\right)(x+\epsilon, t, \epsilon)+\epsilon^{\beta} \mathrm{d} t\right]^{-1}+\mathrm{d} t r(x, t, \epsilon)(\mathrm{d} t)
\end{aligned}
$$

where the new $r$ has still the property that $\|r(., t, \epsilon)(\mathrm{d} t)\|_{\infty} \rightarrow 0$ when $\mathrm{d} t \rightarrow 0$ uniformly for $t \in\left[0, \delta^{\prime}\right]$ if $\delta^{\prime}<\delta(\epsilon)$, but, as always in this proof, without any uniformness in $\epsilon$.

Applying the analog of (27) with $\rho u$ in place of $\rho$, and with the supplementary term $\rho \frac{\partial}{\partial x} \Phi$ from (16), one obtains (from 20, 21) dropping the term $\epsilon^{\beta} \mathrm{d} t$ in the first denominator:

$$
\begin{aligned}
u(x, t+\mathrm{d} t, \epsilon)= & \frac{(\rho u)(x, t+\mathrm{d} t, \epsilon)}{\rho(x, t+\mathrm{d} t, \epsilon)} \\
= & \frac{\frac{\mathrm{d} t}{\epsilon}\left(\rho u u^{+}\right)(x-\epsilon, t, \epsilon)+\left[1-\frac{\mathrm{d} t}{\epsilon}|u|(x, t, \epsilon)\right](\rho u)(x, t, \epsilon)+\frac{\mathrm{d} t}{\epsilon}\left(\rho u u^{-}\right)(x+\epsilon, t, \epsilon)}{\frac{\mathrm{d} t}{\epsilon}\left(\rho u^{+}\right)(x-\epsilon, t, \epsilon)+\left[1-\frac{\mathrm{d} t}{\epsilon}|u|(x, t, \epsilon)\right] \rho(x, t, \epsilon)+\frac{\mathrm{d} t}{\epsilon}\left(\rho u^{-}\right)(x+\epsilon, t, \epsilon)} \\
& -\mathrm{d} t \frac{\rho(x, t, \epsilon)}{\rho(x, t+\mathrm{d} t, \epsilon)} \partial_{x} \Phi(x, t, \epsilon)+\mathrm{d} t r(x, t, \epsilon)(\mathrm{d} t)
\end{aligned}
$$


where the new $r$ has the same property as in (27) for fixed $\epsilon$. For $\mathrm{d} t>0$ small enough, the first term in the second member is a barycentric combination with positive coefficients of $u(x-\epsilon, t, \epsilon), u(x, t, \epsilon)$ and $u(x+\epsilon, t, \epsilon)$ (which are in numerator in factor of $\rho$ inside $\rho u$ ). For fixed $\epsilon$, the quotient $\frac{\rho(x, t+\mathrm{d} t, \epsilon)}{\rho(x, t, \epsilon)}$ tends to 1 when $\mathrm{d} t \rightarrow 0$ uniformly for $t \in\left[0, \delta^{\prime}\right]$ : use (20,21 and 27). It follows from (23) and (28) that

$$
\|u(., t+\mathrm{d} t, \epsilon)\|_{\infty} \leq\|u(., t, \epsilon)\|_{\infty}+\mathrm{d} t \frac{\text { const }}{\epsilon^{3 \alpha}}+\mathrm{d} t\|r(., t, \epsilon)(\mathrm{d} t)\|_{\infty}
$$

where, when $\mathrm{d} t \rightarrow 0$, const $\rightarrow C$ uniformly if $t \in\left[0, \delta^{\prime}\right]$ and where, for fixed $\epsilon, r$ converges uniformly to 0 if $t \in\left[0, \delta^{\prime}\right]$. One obtains the bound (24) from (29) by dividing the interval $[0, t]$ into $n$ intervals $\left[\frac{i t}{n}, \frac{(i+1) t}{n}\right], 0 \leq i \leq n-1$. Application of (29) in each subinterval gives:

$$
\left\|u\left(.,(i+1) \frac{t}{n}, \epsilon\right)\right\|_{\infty} \leq\left\|u\left(., i \frac{t}{n}, \epsilon\right)\right\|_{\infty}+\frac{t}{n} \frac{\text { const }}{\epsilon^{3 \alpha}}+\frac{t}{n} \bar{r}\left(\frac{t}{n}\right),
$$

where const $\rightarrow C$ uniformly if $t \in\left[0, \delta^{\prime}\right]$ when $n \rightarrow \infty$ and $\bar{r}$ is a function of the increment $\mathrm{d} t$, depending on $\epsilon$, such that $\bar{r}(\mathrm{~d} t) \geq \sup _{x, t}|r(x, t, \epsilon)(\mathrm{d} t)|$. One obtains (24) by summing on $i$ and using that $\bar{r}\left(\frac{t}{n}\right) \rightarrow 0$ when $n \rightarrow \infty$, see more details in [11]. One notices that when $n \rightarrow+\infty$, then const can be chosen so as to tend to $C$ and that $\bar{r}$ disappears; then one obtains (24) in which the auxiliary value $\delta^{\prime}$ has disappeared.

Now let us prove inequalities (26). From (15),

$$
\frac{\mathrm{d}}{\mathrm{d} t} \rho(x, t, \epsilon) \geq-\frac{1}{\epsilon}(\rho|u|)(x, t, \epsilon)
$$

since $\rho, u^{+}$and $u^{-}$are positive $(13,20)$. Therefore, from $(24,25)$,

$$
\frac{\mathrm{d}}{\mathrm{d} t} \rho(x, t, \epsilon) \geq-\frac{k(\epsilon)}{\epsilon} \rho(x, t, \epsilon) .
$$

Let $v(x, t, \epsilon)=\rho_{0}^{\epsilon}(x) \exp \left(-\frac{k(\epsilon)}{\epsilon} t\right)$. Then, using assumption (20) to divide by $\rho$,

$$
\frac{\frac{\mathrm{d}}{\mathrm{d} t} \rho}{\rho}(x, t, \epsilon) \geq \frac{\frac{\mathrm{d}}{\mathrm{d} t} v}{v}(x, t, \epsilon)=-\frac{k(\epsilon)}{\epsilon} .
$$

By integration, since $\rho$ and $v$ have same initial condition $\rho_{0}^{\epsilon}(x), \log (\rho) \geq \log (v)$, i.e., $\rho(x, t, \epsilon) \geq$ $v(x, t, \epsilon)$, i.e.,

$$
\rho(x, t, \epsilon) \geq \rho_{0}^{\epsilon}(x) \exp \left(-\frac{k(\epsilon)}{\epsilon} t\right)
$$

which is the left-hand side inequality (26). Now let us prove the right-hand side inequality (26).

From the positiveness of the terms $\rho u^{ \pm}$in (15)

$$
\rho(x, t, \epsilon) \leq \rho_{0}^{\epsilon}(x)+\frac{2}{\epsilon} \int_{0}^{t}\|\rho(., s, \epsilon)\|_{\infty}\|u(., s, \epsilon)\|_{\infty} \mathrm{d} s .
$$

From $(24,25)$, if $t \in[0, \delta(\epsilon)[$

$$
\rho(x, t, \epsilon) \leq\left\|\rho_{0}^{\epsilon}\right\|_{\infty}+\frac{2}{\epsilon} \int_{0}^{t}\|\rho(., s, \epsilon)\|_{\infty} k(\epsilon) \mathrm{d} s .
$$

Since this holds for all $x$

$$
\|\rho(., t, \epsilon)\|_{\infty} \leq\left\|\rho_{0}^{\epsilon}\right\|_{\infty}+\frac{2}{\epsilon} k(\epsilon) \int_{0}^{t}\|\rho(., s, \epsilon)\|_{\infty} \mathrm{d} s .
$$


Gronwall's inequality implies

$$
\|\rho(., t, \epsilon)\|_{\infty} \leq\left\|\rho_{0}^{\epsilon}\right\|_{\infty} \exp \left(\frac{2}{\epsilon} k(\epsilon) t\right)
$$

end of proof of Proposition 1.

For fixed $\epsilon>0$, if $0<\lambda<1$ and

$$
\Omega_{\lambda}:=\left\{(X, Y) \in \mathcal{C}(\mathbb{T})^{2} / \forall x \in \mathbb{T} \quad \lambda<X(x)<\frac{1}{\lambda},|Y(x)|<\frac{1}{\lambda}\right\}
$$

the ODEs (15-17) can be stated in the conventional form

$$
X^{\prime}(t)=F(X(t), Y(t)), Y^{\prime}(t)=G(X(t), Y(t)),
$$

where $F$ and $G$ are the functions from $\cup_{0<\lambda<1} \Omega_{\lambda} \subset(\mathcal{C}(\mathbb{T}))^{2}$ of variables $X=\rho, Y=\rho u$ with values in $\mathcal{C}(\mathbb{T})$ defined by

$$
\begin{aligned}
& F(X(x), Y(x))=\frac{1}{\epsilon}\left[Y^{+}(x-\epsilon)-|Y(x)|+Y^{-}(x+\epsilon)\right]+\epsilon^{\beta}, \\
& G(X(x), Y(x))=\frac{1}{\epsilon}\left[Y \cdot\left(\frac{Y}{X}\right)^{+}(x-\epsilon)-Y\left|\frac{Y}{X}(x)\right|+Y\left(\frac{Y}{X}\right)^{-}(x+\epsilon)\right]-K X(x)\left[\ln \left(X+\epsilon^{N}\right) *\left(\phi_{\epsilon^{\alpha}}\right)^{\prime}\right](x) .
\end{aligned}
$$

For fixed $\epsilon$ the functions $F$ and $G$ are Lipschitz with Lipschitz coefficients uniform in $\Omega_{\lambda}$. Indeed the map $(X, Y): \Omega_{\lambda} \longmapsto \mathcal{C}(\mathbb{T})$ satisfies $\left|\frac{Y_{1}}{X_{1}}-\frac{Y_{2}}{X_{2}}\right| \leq \frac{1}{\lambda^{3}}\left(\left|X_{2}-X_{1}\right|+\left|Y_{2}-Y_{1}\right|\right)$. The maps $X \longmapsto X^{ \pm}$and the translations have Lipschitz coefficients $=1$.

The existence of a unique global solution to (15-18) for fixed $\epsilon$ is obtained from the a priori estimates in Proposition 1 in the proof exposed above in point (4) in the above description of the structure of the proof.

It remains to prove that the solution of the system of ODEs $(15,16)$ complemented by $(17,18)$ provides a weak asymptotic solution for system (10-12) when $\epsilon \rightarrow 0$. To this end, one has to prove that $\forall t>0, \forall \psi \in \mathcal{C}_{c}^{\infty}(\mathbb{R})(36-38)$ below hold when $\epsilon \rightarrow 0$ :

$$
\begin{aligned}
\int_{\mathbb{R}} \frac{\mathrm{d}}{\mathrm{d} t} \rho(x, t, \epsilon) \psi(x) \mathrm{d} x & =\int_{\mathbb{R}}(\rho u)(x, t, \epsilon) \psi^{\prime}(x) \mathrm{d} x+f(\epsilon), \\
\int_{\mathbb{R}} \frac{\mathrm{d}}{\mathrm{d} t}(\rho u)(x, t, \epsilon) \psi(x) \mathrm{d} x & =\int_{\mathbb{R}}\left(\rho u^{2}\right)(x, t, \epsilon) \psi^{\prime}(x) \mathrm{d} x-\int_{\mathbb{R}} \rho(x, t, \epsilon) \partial_{x} \Phi(x, t, \epsilon) \psi(x) \mathrm{d} x+f(\epsilon), \\
\int_{\mathbb{R}} \Phi(x, t, \epsilon) \psi(x) \mathrm{d} x & =\int_{\mathbb{R}} K \ln [\rho(x, t, \epsilon)] \psi(x) \mathrm{d} x+f(\epsilon),
\end{aligned}
$$

where the three different $f(\epsilon)$ tend to 0 when $\epsilon \rightarrow 0$.

The proof of $(36)$ is as follows: from $(14,15,22,24)$, a change of variable and $\frac{\psi(x+\epsilon)-\psi(x)}{\epsilon}=\psi^{\prime}(x)+$ $O_{x}(\epsilon)$

$$
\begin{aligned}
\int_{\mathbb{R}} \frac{\mathrm{d}}{\mathrm{d} t} \rho(x, t, \epsilon) \psi(x) \mathrm{d} x= & \frac{1}{\epsilon} \int_{\mathbb{R}}\left(\rho u^{+}\right)(x, t, \epsilon)[\psi(x+\epsilon)-\psi(x)] \mathrm{d} x-\frac{1}{\epsilon} \int_{\mathbb{R}}\left(\rho u^{-}\right)(x, t, \epsilon) \\
{[\psi(x)-\psi(x-\epsilon)] \mathrm{d} x+\int_{\mathbb{R}} \epsilon^{\beta} \psi(x) \mathrm{d} x=} & \int_{\mathbb{R}}(\rho u)(x, t, \epsilon) \psi^{\prime}(x) \mathrm{d} x+\int_{\text {compact } \subset \mathbb{R}}\left(\rho u^{+}\right)(x, t, \epsilon) O_{x}(\epsilon) \mathrm{d} x \\
& +\int_{\text {compact } \subset \mathbb{R}}\left(\rho u^{-}\right)(x, t, \epsilon) O_{x}(\epsilon) \mathrm{d} x+O\left(\epsilon^{\beta}\right)
\end{aligned}
$$




$$
\begin{aligned}
& =\int_{\mathbb{R}}(\rho u)(x, t, \epsilon) \psi^{\prime}(x) \mathrm{d} x+\left(\text { const }+\frac{\text { const }}{\epsilon^{3 \alpha}} t\right) O(\epsilon)+O\left(\epsilon^{\beta}\right) \\
& =\int_{\mathbb{R}}(\rho u)(x, t, \epsilon) \psi^{\prime}(x) \mathrm{d} x+O\left(\epsilon^{1-3 \alpha}\right)+O\left(\epsilon^{\beta}\right) .
\end{aligned}
$$

This gives (36) if $0<\alpha<\frac{1}{3}$. The proof of (37) is similar since the additional terms $\rho(x, t, \epsilon) \partial_{x} \Phi(x, t, \epsilon)$ in (16) and (37) simplify. One obtains a remainder $\frac{\text { const }}{\epsilon^{6 \alpha}} t O(\epsilon)$ because of one more factor $u$ and the bound (24). Finally one chooses $0<\alpha<\frac{1}{6}$.

To check (38), one has to prove from (18) that

$$
\forall \psi \in \mathcal{C}_{c}^{\infty}(\mathbb{R}) \int_{\mathbb{R}}\left\{\left[\ln \left(\rho(., t, \epsilon)+\epsilon^{N}\right) * \phi_{\epsilon^{\alpha}}\right](x)-\ln [\rho(x, t, \epsilon)]\right\} \psi(x) \mathrm{d} x \rightarrow 0
$$

when $\epsilon \rightarrow 0$. To this end, we share the integral (39) into the two parts $(40,41)$ below and we prove that each of them tends to 0 when $\epsilon \rightarrow 0$. Let

$$
I=\int_{\mathbb{R}}\left\{\left[\ln \left(\rho(., t, \epsilon)+\epsilon^{N}\right) * \phi_{\epsilon^{\alpha}}\right](x)-\ln \left[\rho(x, t, \epsilon)+\epsilon^{N}\right]\right\} \psi(x) \mathrm{d} x
$$

and

$$
J=\int_{\mathbb{R}}\left\{\ln \left[\rho(x, t, \epsilon)+\epsilon^{N}\right]-\ln [\rho(x, t, \epsilon)]\right\} \psi(x) \mathrm{d} x .
$$

Now

$$
\begin{aligned}
I & =\int_{\mathbb{R}}\left\{\ln \left[\rho\left(x-\epsilon^{\alpha} \mu, t, \epsilon\right)+\epsilon^{N}\right]-\ln \left[\rho(x, t, \epsilon)+\epsilon^{N}\right]\right\} \phi(\mu) \psi(x) d \mu \mathrm{d} x \\
& =\int_{\mathbb{R}} \ln \left[\rho(x, t, \epsilon)+\epsilon^{N}\right] \phi(\mu)\left[\psi\left(x+\epsilon^{\alpha} \mu\right)-\psi(x)\right] d \mu \mathrm{d} x .
\end{aligned}
$$

Since $\rho(x, t, \epsilon) \geq 0$ from (26), using (22) in the case $\rho(x, t, \epsilon)>1$ and using $\epsilon^{N}$ in the case $\rho(x, t, \epsilon) \leq 1$, as in the proof of $(23)$, one has $|I| \leq$ const $\ln \left(\frac{1}{\epsilon}\right) \epsilon^{\alpha}$; therefore $I \rightarrow 0$ when $\epsilon \rightarrow 0$.

Now (41) and the mean value theorem give

$$
|J| \leq \epsilon^{N} \frac{1}{\min (\rho)} \text { const }
$$

if $\min (\rho)$ denotes the inf of $\rho(x, t, \epsilon)$ for fixed $t$ and $\epsilon$ when $x$ ranges in $\mathbb{T}$. The problem is to obtain a suitable inf. bound of $\min (\rho)$ when $\epsilon \rightarrow 0$. The term $\epsilon^{\beta}$ in (15) has been introduced for this purpose. Indeed, from (15),

$$
\frac{d \rho}{\mathrm{d} t}(x, t, \epsilon) \geq-\frac{1}{\epsilon} \rho(x, t, \epsilon)\|u(., t, \epsilon)\|_{\infty}+\epsilon^{\beta},
$$

i.e., setting $A:=$ const $\frac{1}{\epsilon^{1+3 \alpha}}$ and $B:=\epsilon^{\beta}$, the fact from (24) that $\|u\|_{\infty} \leq \frac{\text { const }}{\epsilon^{3 \alpha}}$ on any bounded time interval implies that on such interval

$$
\frac{d \rho}{\mathrm{d} t} \geq-A \rho+B
$$

If one considers the ODE $\frac{\mathrm{d} X}{\mathrm{~d} t}(x, t)=-A X(x, t)+B$ with initial condition $X(x, 0)=\rho_{0}^{\epsilon}(x)$, its solution is

$$
X(x, t)=\rho_{0}^{\epsilon}(x) e^{-A t}+\frac{B}{A}\left(1-e^{-A t}\right) .
$$


For fixed $t>0$ and for $\epsilon>0$ small enough, the second term in the right member implies

$$
X(x, t) \geq \frac{B}{2 A}=\operatorname{const}(t) \cdot \epsilon^{1+\beta+3 \alpha},
$$

in which we state the constant as const $(t)$ since this constant depends on $t$ through $(24)$. Note that, in the presence of a void region in the initial condition, $\rho_{0}^{\epsilon}(x)$ can tend to 0 when $\epsilon \rightarrow 0$. Then $X(x, t)$ can tend to 0 when both $t$ and $\epsilon$ tend to 0 . There is no trouble because (38) has to be checked for fixed $t>0$. We have

$$
\rho(x, t, \epsilon) \geq X(x, t)
$$

therefore

$$
\rho(x, t, \epsilon) \geq \operatorname{const}(t) \epsilon^{1+\beta+3 \alpha} .
$$

From (42) $|J| \leq \operatorname{const}(t) \epsilon^{N-1-\beta-3 \alpha}$ and it suffices to choose $\beta+3 \alpha<N-1$ to have that $J \rightarrow 0$ when $\epsilon \rightarrow 0 . \square$ end of proof of Theorem 1 in the 1-D case.

It follows from the proof that the possible presence of void regions, approximated in the initial conditions by $\rho_{0}^{\epsilon}(x) \geq \epsilon \forall x$, does not cause a problem although they appear a priori excluded by the formulation (12). Note also that the presence of $\epsilon$ in denominator in (26) allows concentrations of matter that can even be accepted in initial conditions by choosing $\rho_{0}^{\epsilon}(x) \leq \frac{1}{\epsilon} \forall x$ : The initial condition $\rho_{0}$ can be a positive bounded Radon measure. One could notice that formula (24) allows the possibility of infinite velocity at the limit $\epsilon=0$. This should not be troublesome: The remark that the ideal equations of fluid dynamics could lead to solutions with infinite velocity in certain circumstances has been known since long time [14] section 14.9.1, [21] section 6.6.2, 7.4.3.

Proof of Theorem 1 in the multi-D case. Now we give the 2-D proof (the 3-D proof is identical) in order to convince the reader that the multi-D extension is really straightforward. We adopt some obvious abbreviated notations as often as possible. We state the 2-D equations $(15,16)$ :

$$
\begin{aligned}
\frac{\mathrm{d}}{\mathrm{d} t} \rho(x, y, t, \epsilon)= & \frac{1}{\epsilon}\left[\left(\rho u^{+}\right)(x-\epsilon, y, t, \epsilon)-(\rho|u|)(x, y, t, \epsilon)+\left(\rho u^{-}\right)(x+\epsilon, y, t, \epsilon)\right. \\
& \left.+\left(\rho v^{+}\right)(x, y-\epsilon, t, \epsilon)-(\rho|v|)(x, y, t, \epsilon)+\left(\rho v^{-}\right)(x, y+\epsilon, t, \epsilon)\right]+\epsilon^{\beta},
\end{aligned}
$$

for some $\beta>0$ to be made precise later,

$$
\begin{aligned}
\frac{\mathrm{d}}{\mathrm{d} t}(\rho u)(x, y, t, \epsilon)= & \frac{1}{\epsilon}\left[\left(\rho u u^{+}\right)(x-\epsilon, y, t, \epsilon)-(\rho u|u|)(x, y, t, \epsilon)+\left(\rho u u^{-}\right)(x+\epsilon, y, t, \epsilon)\right. \\
& \left.+\left(\rho u v^{+}\right)(x, y-\epsilon, t, \epsilon)-(\rho u|v|)(x, y, t, \epsilon)+\left(\rho u v^{-}\right)(x, y+\epsilon, t, \epsilon)\right] \\
& -\rho(x, y, t, \epsilon) \partial_{x} \Phi(x, y, t, \epsilon),
\end{aligned}
$$

and a similar Euler equation in which $\rho u$ is replaced by $\rho v$ and $\partial_{x} \Phi$ by $\partial_{y} \Phi$. $\Phi$ is again given by (18) with the difference that now $\phi \in \mathcal{C}_{c}^{\infty}\left(\mathbb{R}^{2}\right)$ and $\phi_{\mu}(x, y)=\frac{1}{\mu^{2}} \phi\left(\frac{x}{\mu}, \frac{y}{\mu}\right)$ : This will change $\epsilon^{3 \alpha}$ into $\epsilon^{4 \alpha}$ in (23-25). The system of three ODEs in $\rho, \rho u$ and $\rho v$ gives a local solution $\left[0, \delta(\epsilon)\left[\longmapsto\left(\mathcal{C}\left(\mathbb{T}^{2}\right)\right)^{3}, t \longmapsto(\rho, \rho u, \rho v)\right.\right.$ for which one has again $(20,21)$ stated in $\rho, u$ and $v$ from the choice of the approximation of the initial condition in case of void regions.

Concerning the a priori estimates in Proposition 1, formula (22) is unchanged by integration over $\mathbb{T}^{2}$ involving $\rho u^{ \pm}$and $\rho v^{ \pm}$since same simplifications occur. In $\partial_{x} \Phi$ one has now a function $\phi$ of two variables and $\frac{1}{\epsilon^{3 \alpha}}$ instead of $\frac{1}{\epsilon^{2 \alpha}}$; therefore, in (23), $\frac{1}{\epsilon^{4 \alpha}}$ replaces $\frac{1}{\epsilon^{3 \alpha}}$. Formula (27) involves now $\rho u^{ \pm}, \rho v^{ \pm}$ and $1-\frac{\mathrm{d} t}{\epsilon}|u|>0$ is replaced by $1-\frac{\mathrm{d} t}{\epsilon}|u|-\frac{\mathrm{d} t}{\epsilon}|v|>0$ with again same domination for fixed $\epsilon$ provided $\mathrm{d} t>0$ small enough depending on $\epsilon$. The second line of $(28)$ involves now $\rho u u^{+}(x-\epsilon, y, t, \epsilon), \rho u v^{+}(x, y-$ $\epsilon, t, \epsilon),\left(1-\frac{\mathrm{d} t}{\epsilon}|u|-\frac{\mathrm{d} t}{\epsilon}|v|\right) \rho u, \rho u u^{-}, \rho u v^{-}$and similar denominator where $\rho u$ is replaced by $\rho$ : One has the same barycentric combination of $\rho u$ in numerator and $\rho$ in denominator, which yields (29) with $4 \alpha$ in place of $3 \alpha$ because of $\partial_{x} \Phi$. To prove (26), one has the two terms $\rho|u|$ and $\rho|v|$ in (44) therefore $2 k(\epsilon)$ in place of $k(\epsilon)$ in (30). Similarly in (33), one has $\frac{4}{\epsilon}$ in place of $\frac{2}{\epsilon}$ therefore also in (34). 
The Lipschitz property of the new $F(X, Y, Z), G(X, Y, Z), X=\rho, Y=\rho u, Z=\rho v$ is unchanged. Therefore, the proof of global solution is unchanged. The proof of the 2-D analog of (36) simply involves supplementary terms $\rho v^{ \pm}$that correspond to $\partial_{y}(\rho v)$. In $(39,40)$ one has a convolution in two variables $x, y$; therefore, one has $\psi\left(x+\epsilon^{\alpha} \mu, y+\epsilon^{\alpha} \nu\right)-\psi(x, y)$ after (41). (42) is unchanged with $\min (\rho)=i n f_{x, y} \rho(x, y, t, \epsilon)$. From (44) $A$ becomes 2 const $\frac{1}{\epsilon^{1+4 \alpha}}$. Therefore, Theorem 1 holds in 2 -D with $\alpha<\frac{1}{8}$ and $4 \alpha+\beta<N-1$. In 3 -D one has no more than the slight change to $\frac{1}{10}$ and $5 \alpha$ in these inequalities. $\square$ end of proof of Theorem 1 in the multi-D case.

Numerical confirmations The discretization in time is standard from numerical schemes for ODEs: For fixed $\epsilon>0$, the Lipschitz properties of the ODEs $(15,16)$ permit to prove convergence of the explicit Euler order one method.

All tests are interpreted in the periodic case as being done in each period with periods large enough to avoid interactions between periods during the time interval under consideration. The discretization in space is as follows: The space $\mathbb{R}^{n}$ is discretized by cells of length $\epsilon$ in each direction. The physical variables are constant in the cells. Numerical tests from the explicit Euler order one method and the RK4 Runge-Kutta method for the solution of the ODEs $(15,16)$ have shown that our construction always gives the correct solutions, even in presence of void regions, as this is the case in Figs. 1, 2 and 5, and shocks as this is the case in Fig. 5 top and bottom.

In Fig. 1 we present a demanding test from [5] where an explicit solution is given. The numerical solution coincides with the explicit solution which consists only of a rarefaction wave [5]. It is the Riemann problem $\rho_{l}=0, \rho_{r}=1, u_{l}=0=u_{r}$ at time $T=0.5$ and with $K=0.04$, which has been considered in [5] p. 154 and p. 157: This test is difficult due to the void region on the left in the initial conditions. The test has been done with $\mathrm{d} t=0.00002, \epsilon=0.001,2000$ space steps.

A striking fact is that one observes that all the technical ingredients $\beta, N, \phi_{\epsilon^{\alpha}}, \rho_{0}^{\epsilon}>0$ in the construction of the weak asymptotic solution of Sect. 2 are really indispensible numerically: In the tests in Figs. 1 and 2 , the numerical method fails to give a result if one of these ingredients is not taken into account. In Fig. 1 one has used $\rho_{l}=10^{-6}$ since, by construction, we approximate void regions by regions with very small density $\beta=10, N=2$ and an averaging on three cells to express the convolution in the state law (18) with coefficients $0.3,0.4$ and 0.3 . This averaging consists in replacing a quantity $q_{i}\left(=\ln \left(\rho_{i}\right)+\epsilon^{N}\right.$ in (18)), $i \in \mathbb{Z}$, by $0.3 q_{i-1}+0.4 q_{i}+0.3 q_{i+1}$. When $\epsilon$ becomes very small, one observes the need to involve more cells in the convolution (from the appearance of defects such as oscillations) as justified from the fact the number of cells involved in the convolution (18) is proportional to $\frac{\epsilon^{\alpha}}{\epsilon}$. We have observed numerically that the results are independent of the arbitrariness in a choice of these parameters provided they satisfy requirements such as those in Theorem 1.

\section{Convergence to the analytic solution}

In this section, we present a slight modification in the construction of the weak asymptotic solution in Sect. 2 which permits to prove easily that when the initial data are analytic the weak asymptotic solution gives the classical analytic solutions. This slight modification appears also useful in some numerical tests to suppress oscillations and various numerical defects.

Let us consider a function $v(u),|u|<v(u)<|u|+\nu \forall u \in \mathbb{R}$, where $\nu>0$ is fixed, and where the function $v$ is analytic, for instance $v(u)=\left(u^{2}+\nu^{2}\right)^{\frac{1}{2}}$ that we will use from now on. Let us define $u^{ \pm}$by

$$
u^{+}-u^{-}=u, u^{+}+u^{-}=v(u)
$$

instead of $u^{+}+u^{-}=|u|$ in $(13,14)$. This gives

$$
u^{+}=\frac{v(u)+u}{2}=\frac{v(u)-|u|}{2}+\frac{|u|+u}{2}, u^{-}=\frac{v(u)-u}{2}=\frac{v(u)-|u|}{2}+\frac{|u|-u}{2}
$$



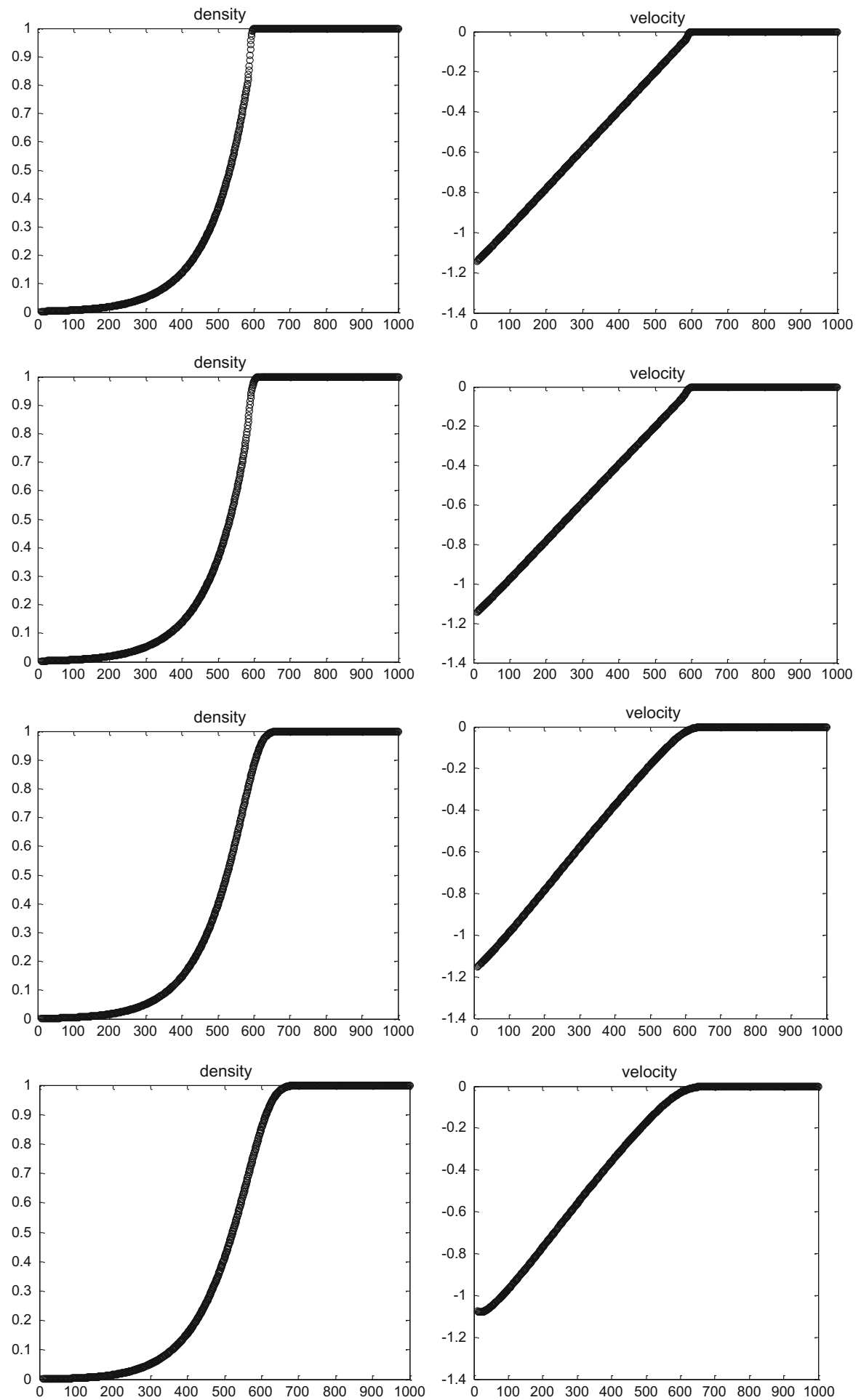

FIG. 2. The test of Fig. 1 with variable values of the parameter $\nu=0.001,0.1,1,2$ from top to bottom. The test of Fig. 1 corresponds to $\nu=0$ 
i.e., $u^{+}$and $u^{-}$are larger by the quantity $\frac{v(u)-|u|}{2}$ than their previous values considered in Sect. 2 . Now let us consider the ODE

$$
\frac{\mathrm{d}}{\mathrm{d} t} \rho(x, t, \epsilon)=\frac{1}{\epsilon}\left[\left(\rho u^{+}\right)(x-\epsilon, t, \epsilon)-(\rho v(u))(x, t, \epsilon)+\left(\rho u^{-}\right)(x+\epsilon, t, \epsilon)\right]+\epsilon^{\beta}
$$

instead of (15), with $u^{ \pm}$and $v(u)$ defined in (46), as well as a similar use of the new values $u^{ \pm}$and $v(u)$ in (16) giving $\frac{\mathrm{d}}{\mathrm{d} t}(\rho u)$.

First, one can check that the proof of weak asymptotic solution in Sect. 2 holds without change: This is based on the fact that the presence of any fixed value $\nu>0$ does not affect significantly the bounds and that one uses only the formula $u=u^{+}-u^{-}$once one has replaced $v(u)$ by $u^{+}+u^{-}$in (48) and in the ODE satisfied by $\rho u$. Second, numerical tests (Fig. 2) show that the presence of a fixed $\nu>0$ affects the numerical results in the same way as a viscosity. $(47,48)$

If we state $D(u)=\frac{v(u)-|u|}{2}$ and $\bar{u}^{ \pm}=\frac{|u| \pm u}{2}$, i.e., $\bar{u}^{ \pm}$are the values $u^{ \pm}$used in Sect. 2, we obtain from

$$
\begin{aligned}
\frac{\mathrm{d}}{\mathrm{d} t} \rho(x, t, \epsilon) & \\
= & \frac{1}{\epsilon}\left[\left(\rho \bar{u}^{+}\right)(x-\epsilon, t, \epsilon)-\left(\rho \bar{u}^{+}\right)(x, t, \epsilon)-\left(\rho \bar{u}^{-}\right)(x, t, \epsilon)+\left(\rho \bar{u}^{-}\right)(x+\epsilon, t, \epsilon)\right] \\
& +\epsilon^{\beta}+\frac{1}{\epsilon}[(\rho D(u))(x-\epsilon, t, \epsilon)-2(\rho D(u))(x, t, \epsilon)+(\rho D(u))(x+\epsilon, t, \epsilon)] .
\end{aligned}
$$

The two first terms in the second member give the formula used in Sect. 2. In the last term, one recognizes the classical discretization of $\epsilon \partial_{x x}(\rho D(u))$; therefore, this term is some kind of vanishing viscosity, which explains the results observed in Fig. 2.

Numerical confirmation In Fig. 2 the discretization is exactly the same as in Sect. 2. The difference is the use of $v(u)=\left(u^{2}+\nu^{2}\right)^{\frac{1}{2}}$ instead of $|u|$. We observe that $\nu$ has the same influence as a viscosity coefficient: For $\nu=0.001$ (top panel) and $\nu=0.1$, one observes the exact solution; for $\nu=1$, one observes some viscosity effects which become important for $\nu=2$ (bottom panel).

Coherence with the analytic solutions The Cauchy-Kovalevskaya theorem is the classical existenceuniqueness result that applies in general to the systems of nonlinear analytic first-order equations such as those under consideration in this paper. Although it is unsatisfactory in practice due to its limitation to analytic solutions, it should be proved that the approximate solutions we construct converge to the analytic solution when the Cauchy-Kovalevskaya theorem applies. This is the aim of the sequel of this section.

From the replacement of $|u|$ by an analytic regularization $v(u)$, we prove that our approximate solutions tend to the classical analytic solution at the limit $\epsilon \rightarrow 0$, which was proved in [10] in a linear case when the function $u$ has a fixed sign to avoid the singularity of the function absolute value at 0 . The proof is given in the 1-D case since the multi-dimensional case is identical. The proof consists in applying the abstract nonlinear Cauchy-Kovalevskaya theorem of Nirenberg and Nishida [28] for each $\epsilon>0$ small enough, with the observation that the results are uniform in $\epsilon$, which permits to pass to the limit $\epsilon \rightarrow 0$ using the theory of normal families of holomorphic functions.

Recall of an abstract nonlinear Cauchy-Kovalevskaya theorem [28] By definition a scale of Banach spaces is a family of Banach spaces $\left(E_{s}\right)_{s}, 0<s \leq s_{0}$, such that $\left.\left.\forall s, s^{\prime} \in\right] 0, s_{0}\right], s>s^{\prime} \Rightarrow E_{s} \subset E_{s^{\prime}}$ with inclusion of norm $\leq 1$. Let $v_{0} \in E_{s_{0}}$ be given. If $R>0$, we denote by $B_{s}\left(v_{0}, R\right)$ the open ball in the Banach space $E_{s}$ of center $v_{0}$ and radius $R$.

Let $\left(E_{s}\right)_{0<s \leq s_{0}}$ be a scale of complex Banach spaces. Consider the Cauchy problem

$$
\frac{\mathrm{d} v}{\mathrm{~d} t}(t)=G(v(t)), \quad t \in \mathbb{C}, v(0)=v_{0}
$$

Assume the existence of $R>0, C>0, K>0$ such that properties i) and ii) hold 
i $\forall s, s^{\prime} / 0<s^{\prime}<s<s_{0}$ the map $u \longmapsto G(u)$ is holomorphic from $B_{s}\left(v_{0}, R\right)$ into $E_{s^{\prime}}$ and satisfies a Lipschitz property in the sense

$$
\forall u \in B_{s}\left(v_{0}, R\right) \quad\left\|G^{\prime}(u)\right\|_{L\left(E_{s}, E_{s^{\prime}}\right)} \leq \frac{C}{s-s^{\prime}} .
$$

ii $\forall s<s_{0} \quad G\left(v_{0}\right) \in E_{s}$ and

$$
\left\|G\left(v_{0}\right)\right\|_{s} \leq \frac{K}{s_{0}-s}
$$

The abstract Cauchy-Kovalevskaya theorem states: then $\exists$ a number $a>0$ and a unique holomorphic function $t \longmapsto v(t)$ which $\forall s<s_{0}$ maps $\left\{t \in \mathbb{C} /|t|<a\left(s_{0}-s\right)\right\}$ into $B_{s}\left(v_{0}, R\right)$ and is solution of (50).

The domain of $v$ depends on its range through the number $s$ : One understands that the functions $v$ relative to various values of $s$ stick together. The proof is a holomorphic form of the theorem in [28], setting there $u=v-v_{0}, F(u, t)=G(v)$. From the mean value theorem property (51) implies the Lipschitz property stated in [28] if $u, v \in B_{s}\left(v_{0}, R\right)$ :

$$
\|G(u)-G(v)\|_{s^{\prime}} \leq \frac{C}{s-s^{\prime}}\|u-v\|_{s} \forall u, v \in B_{s}\left(v_{0}, R\right) .
$$

The method of proof is the iteration method with adequate bounds, see [28] in which it is proved that the successive iterates lie in $B_{s}\left(v_{0}, R\right)$. The number $a>0$ depends only on $s_{0}, R, C, K$ (formulas $13 \mathrm{p}$. 630 and end of p. 632 in [28]).

Now, from this abstract Cauchy-Kovalevskaya theorem, we can prove the following coherence result.

Proposition 2. We consider real-valued analytic initial data $\left(\rho_{0}, \rho_{0} u_{0}\right)$, independent of $\epsilon$, satisfying $\rho_{0}(x)>$ $0 \forall x \in \mathbb{T}$. Then if $|t|$ is small enough, the solution of (15-18) with the modification (48) tends to the classical analytic solution when $\epsilon \rightarrow 0$.

Proof. We denote by $\mathcal{H}(\mathbb{T} \times]-s, s[)$, respectively, $\mathcal{C}(\mathbb{T} \times[-s, s])$, the spaces of all holomorphic, respectively, continuous, functions $f=f(z), z=x+i y, x \in \mathbb{T}, y \in \mathbb{R}$ on the open strip $\mathbb{T} \times]-s, s[\subset \mathbb{C}$, respectively, the closed strip $\mathbb{T} \times[-s, s]$. For fixed $\epsilon$, we apply the abstract Cauchy-Kovalevskaya theorem above with the classical scale of Banach spaces

$$
E_{s}=\left\{(f, g) \in[\mathcal{H}(\mathbb{T} \times]-s, s[) \cap \mathcal{C}(\mathbb{T} \times[-s, s])]^{2}\right\}
$$

equipped with the norm

$$
\|(f, g)\|_{s}=\sup _{x \in \mathbb{T},|y| \leq s}(|f(z)|,|g(z)|) .
$$

The real number $s_{0}>0$ is chosen small enough so that the initial conditions $v_{0}^{\epsilon}:=v_{0}=\left(\rho_{0}, \rho_{0} u_{0}\right)$, which are independent on $\epsilon$ and are holomorphic extensions of the given real analytic initial data, are elements of the space $E_{s_{0}}$.

Since we assume $\rho_{0}(x)>0 \forall x \in \mathbb{T}$ then $\min _{x \in \mathbb{T}} \rho_{0}(x)>0$ and therefore $\min _{x \in \mathbb{T},|y| \text { small }}\left|\rho_{0}(z)\right|>0$, one can choose $R>0$ and $s_{0}>0$ small enough so that if $(\rho, \rho u) \in B_{s_{0}}\left(v_{0}, R\right)$ then $\exists b>0 /|\rho(z)|>$ $b \forall z=x+i y, x \in \mathbb{T},|y| \leq s_{0}$, which permits division by $\rho$ at each step in the iterations of the proof in [28] (since the iterates remain in $B_{s}\left(v_{0}, R\right), s<s_{0}$ ) to obtain $u=\frac{\rho u}{\rho}$.

We will also choose $R>0$ and $s_{0}>0$ small enough so that, for a fixed $\nu>0$ in the definition of $v(u)=\left(u^{2}+\nu^{2}\right)^{\frac{1}{2}}$, then $\forall(\rho, \rho u) \in B_{s_{0}}\left(v_{0}, R\right)$ the function $v$ is defined on the set $\frac{\rho u}{\rho}(\mathbb{T} \times]-s_{0}, s_{0}[)$ and bounded there, i.e., the set $\left\{\left(\frac{\rho u}{\rho}(x+i y)\right)^{2}+\nu^{2}\right\}_{x \in \mathbb{T},|y| \leq s_{0}}$ should remain at a strictly positive distance of the negative real demi-axis so that its complex square root could be defined as usual. This is possible by continuity since $\rho_{0}(x)$ and $u_{0}(x)$ are real-valued $\forall x \in \mathbb{T}$ and since $\mathbb{T}$ is compact.

Then for any fixed $\epsilon>0$ small enough, we consider the map $G_{\epsilon}$ defined from (47, 48 and (16) modified by use of $v(u))$ on $B_{s}\left(v_{0}, R\right) \forall s<s_{0}$ with values in $E_{s^{\prime}}, s^{\prime}<s$ by $u=\frac{\rho u}{\rho}$ and

$$
G_{\epsilon}:\left[z \longmapsto ( \rho ( z ) , ( \rho u ) ( z ) ] \in B _ { s } ( v _ { 0 } , R ) \longmapsto \left[\left[z \longmapsto \left\{\frac { 1 } { \epsilon } \left[\left(\rho \cdot u^{+}\right)(z-\epsilon)\right.\right.\right.\right.\right.
$$




$$
\begin{aligned}
& \left.-\left(\rho \cdot\left(u^{+}+u^{-}\right)\right)(z)+\left(\rho \cdot u^{-}\right)(z+\epsilon)\right]+\epsilon^{\beta}, \frac{1}{\epsilon}\left[\left(\rho u \cdot u^{+}\right)(z-\epsilon)-\left(\rho u \cdot\left(u^{+}+u^{-}\right)\right)(z)\right. \\
& \left.\left.\left.\left.+\left(\rho u \cdot u^{-}\right)(z+\epsilon)\right]-\rho(z) \partial_{x} \Phi(z)\right\}\right]\right] \in E_{s^{\prime}} .
\end{aligned}
$$

For fixed $\epsilon$, we apply the abstract Cauchy-Kovalevskaya theorem with the analytic initial condition $v_{0}=\left(\rho_{0}, \rho_{0} u_{0}\right)$ independent on $\epsilon$ and with the map $G_{\epsilon}$. We will obtain a solution $v^{\epsilon}=\left(\rho^{\epsilon},(\rho u)^{\epsilon}\right)$ defined for $|t|<a\left(s_{0}-s\right)$ (with $a$ independent on $\epsilon$, see below) taking values in $B_{s}\left(v_{0}, R\right) \forall s<s_{0}$ (the domain of $v^{\epsilon}$ depends of $s$ : The same abuse of language has been done in the statement of the theorem above). The key of the proof lies in that the assumptions of the abstract Cauchy-Kovalevskaya theorem are satisfied uniformly in $\epsilon$; therefore, the properties (domains and bounds, in particular the number $a$ ) of the solution will be also independent of $\epsilon$ since the proof of [28] is done by iteration from bounds on the successive iterates depending on the domains and bounds in the assumptions.

The verification of the assumptions $(51,52)$ is based on the mean value theorem and Cauchy's inequalities for holomorphic functions, [37] p. 145. This can be checked easily: For simplification if $G_{\epsilon}(\rho, \rho u)$ were the single function $z \longmapsto \frac{\rho u(z-\epsilon)-\rho u(z)}{\epsilon}$ then $\frac{\partial}{\partial(\rho u)} G_{\epsilon}(\rho, \rho u) . w$ would be the function $z \longmapsto \frac{w(z-\epsilon)-w(z)}{\epsilon}$ which is bounded as $(w)^{\prime}(z-\theta(\epsilon, z) \epsilon), 0<\theta(\epsilon, z)<1$; therefore, this amounts to a derivative, and Cauchy's formula shows that $\frac{\partial}{\partial(\rho u)} G_{\epsilon}(\rho, \rho u)$ would map $E_{s}$ into $E_{s^{\prime}}, s>s^{\prime}$ with operator norm $\leq \frac{1}{s-s^{\prime}}$ uniformly in $\epsilon$. The detailed formulas are more complicated due to the presence of $u=\frac{\rho u}{\rho}$ in $u^{ \pm}$inside the formula of $G_{\epsilon}$ and from the definition of $u^{ \pm}$in (46) through the function $v$, but the verification is similar.

To clarify, we give the formulas in the particular case $u \geq 0$ and $v(u)=|u|=u$ which implies $u^{+}=u$ and $u^{-}=0$ and avoids longer formulas. Then from (56) if log denotes the usual complex logarithm

$$
G_{\epsilon}(\rho, \rho u)=z \longmapsto\left\{\frac{\rho u(z-\epsilon)-\rho u(z)}{\epsilon}+\epsilon^{\beta}, \frac{\rho u^{2}(z-\epsilon)-\rho u^{2}(z)}{\epsilon}-\rho(z) \partial_{x} \Phi(z)\right\}
$$

where $\Phi(z)=K\left[\log \left(\rho()+.\epsilon^{N}\right) * \phi_{\epsilon^{\alpha}}\right](z)$ from $(18)$.

Set $X=\rho, Y=\rho u$, then

$$
G_{\epsilon}(X, Y)=z \longmapsto\left\{\frac{Y(z-\epsilon)-Y(z)}{\epsilon}+\epsilon^{\beta}, \frac{\frac{Y^{2}}{X}(z-\epsilon)-\frac{Y^{2}}{X}(z)}{\epsilon}-K X(z)\left[\log \left(X+\epsilon^{N}\right) *\left(\phi_{\epsilon^{\alpha}}\right)^{\prime}\right](z)\right\} .
$$

Therefore

$$
\begin{aligned}
D G_{\epsilon}(X, Y) \cdot\left(w_{1}, w_{2}\right)= & z \longmapsto\left\{\frac{w_{2}(z-\epsilon)-w_{2}(z)}{\epsilon}, \frac{\frac{2 Y w_{2}}{X}(z-\epsilon)-\frac{2 Y w_{2}}{X}(z)}{\epsilon}-\frac{\frac{Y^{2} w_{1}}{X^{2}}(z-\epsilon)-\frac{Y^{2} w_{1}}{X^{2}}(z)}{\epsilon}\right. \\
& \left.-K w_{1}(z)\left[\log \left(X+\epsilon^{N}\right) *\left(\phi_{\epsilon^{\alpha}}\right)^{\prime}\right](z)-K X(z)\left[\frac{w_{1}}{X+\epsilon^{N}} *\left(\phi_{\epsilon^{\alpha}}\right)^{\prime}\right](z)\right\} .
\end{aligned}
$$

If $(X, Y) \in B_{s}\left(v_{0}, R\right) \subset E_{s}$, then the values $X(x+i y), Y(x+i y)$ are defined for $x \in \mathbb{T},|y|<s$ and are bounded in sup norm by $\left\|v_{0}\right\|_{s}+R$. Further $X(x+i y)>b>0$; the terms $\epsilon^{N}$ and $\epsilon^{\beta}$ in $(58,59)$ are not used to check the validity of $(51,52)$ for $G_{\epsilon}$ uniformly in $\epsilon$.

The abstract Cauchy-Kovalevskaya theorem asserts the existence of a solution $v^{\epsilon}: t \longmapsto$ $\left(\rho^{\epsilon}(., t),(\rho u)^{\epsilon}(., t)\right) \in B_{s}\left(v_{0}, R\right)$ to the ODEs (48) and (16 modified) defined on a domain of time $\left\{|t|<a\left(s_{0}-s\right)\right\}$ which is independent of $\epsilon$ since domains and bounds on the data are uniform in $\epsilon$. Since the domains and bounds of the solutions are independent of $\epsilon$ the family $\left(v^{\epsilon}\right)$ is a normal family of holomorphic functions on $\left.\left\{|t|<a\left(s_{0}-s\right)\right\} \times \mathbb{T} \times\right]-s,+s\left[\forall s\right.$. Fix a value of $s$. From any sequence $\left(v^{\epsilon_{n}}\right)_{n}$, one can extract a convergent subsequence. This subsequence converges to the classical analytic solution of the system of PDEs $(10,11)$. Therefore, the whole family $\left(v^{\epsilon}\right)$ converges to the classical analytic solution. 
Now we will consider two systems very close to the system of isothermal gases: the system of isentropic gases and the shallow water equations. The proofs are nearly identical so we only sketch them in 1-D. The multi-D proofs are identical to the 1-D proofs as in Sect. 2.

\section{Sequence of approximate solutions to the system of isentropic gas equations}

The system of isentropic gas Eqs. $(2,3,4,6)$ is stated in 1-D in the form

$$
\begin{aligned}
& \partial_{t} \rho+\partial_{x}(\rho u)=0, \\
& \partial_{t}(\rho u)+\partial_{x}\left(\rho u^{2}\right)+\rho \partial_{x} \Phi=0, \\
& \Phi=\frac{K \gamma}{\gamma-1} \rho^{\gamma-1}, K=\text { const } \geq 0,1<\gamma \leq 2,
\end{aligned}
$$

therefore $\rho \partial_{x} \Phi=K \partial_{x}\left(\rho^{\gamma}\right)=p_{x}$ if $p=K \rho^{\gamma}$. We state the system of ODEs as follows: We state the ODE (15) without the $\epsilon^{\beta}$ term which is no more needed because the state law (62) is simpler than the state law (12) for our proof, i.e.,

$$
\frac{\mathrm{d}}{\mathrm{d} t} \rho(x, t, \epsilon)=\frac{1}{\epsilon}\left[\left(\rho u^{+}\right)(x-\epsilon, t, \epsilon)-(\rho|u|)(x, t, \epsilon)+\left(\rho u^{-}\right)(x+\epsilon, t, \epsilon)\right],
$$

(in which $u^{ \pm}$are given by $(13,14)$, but could also be given by $(46,47)$ with the replacement of $|u|$ by $v(u))$. Then we state the ODE (16), the formula (17), and we replace the formula (18) by

$$
\Phi(x, t, \epsilon)=\frac{K \gamma}{\gamma-1}\left[(\rho(., t, \epsilon))^{\gamma-1} * \phi_{\epsilon^{\alpha}}\right](x) .
$$

in which the term $\epsilon^{N}$ in (18) is no longer needed.

As usual the convolution in (64) is justified by the fact that the state law is obtained from measurements which are always done in a space region which cannot be extremely small (explanations after (18)). As in Sect. 2, we assume initial conditions $\rho_{0}^{\epsilon}$ and $u_{0}^{\epsilon}$ defined on $\mathbb{T}$ with same properties $(20,21)$. To obtain the a priori inequalities we assume (19-21). Then we obtain the same a priori inequalities as in Proposition 1:

the statements (22 without the $\epsilon^{\beta}$ term), (23, 24, 25 with $2 \alpha$ in place of $3 \alpha$ ) and (26) hold as in Proposition 1.

Proof. The proof of (22) in the present case is identical to the proof in Proposition 1 without the $\epsilon^{\beta}$ term. For the proof of (23) here we have from (64)

$$
\partial_{x} \Phi(x, t, \epsilon)=\frac{K \gamma}{\gamma-1} \int_{\mathbb{R}}[\rho(x-y, t, \epsilon)]^{\gamma-1} \frac{1}{\epsilon^{2 \alpha}} \phi^{\prime}\left(\frac{y}{\epsilon^{\alpha}}\right) \mathrm{d} y .
$$

Since $0<\gamma-1 \leq 1$ and since from $(22) \rho \in L^{1}(\mathbb{T})$ with $\|\rho\|_{L^{1}(\mathbb{T})}$ independent of $t$ and $\epsilon$, then a fortiori $\rho^{\gamma-1} \in L^{1}(\mathbb{T})$ with bounds independent of $t$ and $\epsilon$. Therefore

$$
\left|\partial_{x} \Phi(x, t, \epsilon)\right| \leq \frac{K \gamma}{\gamma-1}\left\|\rho^{\gamma-1}\right\|_{L^{1}(x-\operatorname{supp} \phi)} \frac{1}{\epsilon^{2 \alpha}}\left\|\phi^{\prime}\right\|_{\infty} \leq \frac{\text { const }}{\epsilon^{2 \alpha}} .
$$

The proofs of (24) and (26) in the present context are identical to those in Proposition 1.

From the a priori estimates one obtains existence and uniqueness of a global solution in time $t \in[0,+\infty[$ and in space $x \in \mathbb{T}$. The analogs of $(36,37)$ are proved as in Sect. 2 ; here it suffices to have $\alpha<\frac{1}{4}$ since (23) is stated with $2 \alpha$. For the state law (62), we have to prove that $\forall \psi \in \mathcal{C}_{c}^{\infty}(\mathbb{R}), \forall t$

$$
\int_{\mathbb{R}}\left[\Phi(x, t, \epsilon)-\frac{K \gamma}{\gamma-1}(\rho(x, t, \epsilon))^{\gamma-1}\right] \psi(x) \mathrm{d} x \rightarrow 0
$$



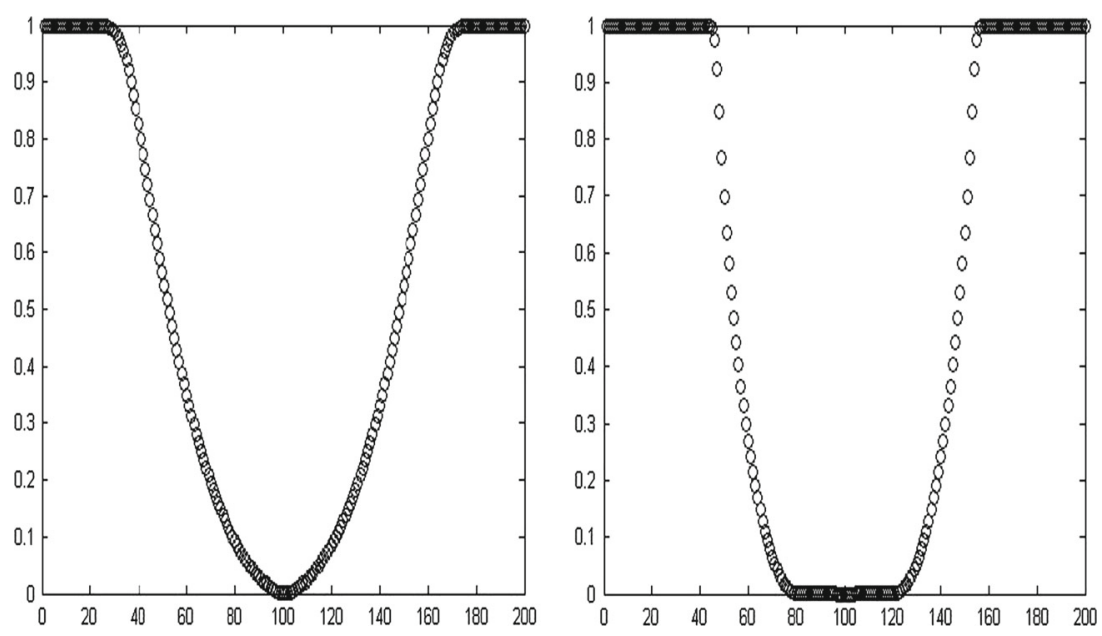

FIG. 3. The observed result in the left panel for which the Mach number $M=\frac{1.3}{\gamma-1}<\frac{2}{\gamma-1}$ suggests absence of void region. The observed result in the right panel for which $M=\frac{2.7}{\gamma-1}>\frac{2}{\gamma-1}$ suggests a void region. This is in agreement with the explicit results in [19] for which the critical value of $M$ is $\frac{2}{\gamma-1}$

when $\epsilon \rightarrow 0$ where $\Phi(x, t, \epsilon)$ is given by (64). This integral is equal to

$$
\begin{aligned}
& \frac{K \gamma}{\gamma-1} \int_{x}\left[\int_{y}(\rho(x-y, t, \epsilon))^{\gamma-1} \frac{1}{\epsilon^{\alpha}} \phi\left(\frac{y}{\epsilon^{\alpha}}\right) \mathrm{d} y-(\rho(x, t, \epsilon))^{\gamma-1}\right] \psi(x) \mathrm{d} x \\
& =\frac{K \gamma}{\gamma-1} \int_{x, \mu}\left[\left(\rho\left(x-\epsilon^{\alpha} \mu, t, \epsilon\right)\right)^{\gamma-1}-(\rho(x, t, \epsilon))^{\gamma-1}\right] \phi(\mu) \psi(x) d \mu \mathrm{d} x \\
& =\frac{K \gamma}{\gamma-1} \int_{x, \mu}(\rho(x, t, \epsilon))^{\gamma-1} \phi(\mu)\left[\psi\left(x+\epsilon^{\alpha} \mu\right)-\psi(x)\right] d \mu \mathrm{d} x=O\left(\epsilon^{\alpha}\right)
\end{aligned}
$$

where the integrals are taken over $\mathbb{R}$ from the $L^{1}(\mathbb{T})$ integrability of $\rho$ and since $0<\gamma-1 \leq 1$ which permits integrability of $\rho^{\gamma-1}$. The 2-D and 3-D proofs are straightforward adaptations of the 1-D proof as in Sect. 2.

Finally we have obtained: Let the initial conditions $\rho_{0} \in L^{1}(\mathbb{T})$, more generally a positive bounded Radon measure, $u_{0} \in L^{\infty}(\mathbb{T})$ and $\rho_{0} \geq 0$. Approximate the initial conditions by a family $\rho_{0}^{\epsilon}, u_{0}^{\epsilon} \in$ $\mathcal{C}(\mathbb{T}), \rho_{0}^{\epsilon}(x)>0 \forall x,\left\|\rho_{0}-\rho_{0}^{\epsilon}\right\|_{L^{1}(\mathbb{T})} \rightarrow 0$ and $\left|\left\|u_{0}\right\|_{\infty}-\left\|u_{0}^{\epsilon}\right\|_{\infty}\right| \rightarrow 0$ when $\epsilon \rightarrow 0$, then if $\alpha<\frac{1}{4}:$

Theorem 2. The solution of the system of ODEs $(16,17,63,64)$ with initial conditions $\left(\rho_{0}^{\epsilon}, u_{0}^{\epsilon}\right)$ provides a weak asymptotic solution (1) for the 1-D isentropic gas equations (60-62), which is global in time $t \in[0,+\infty[$ and in space $x \in \mathbb{T}$. The result holds as well in 2-D and 3-D if $\alpha>0$ is small enough.

In [19] Sect. 4 the authors consider the 1-D Riemann problem $\rho_{l}=\rho_{r}=1, u_{l}=-1, u_{r}=1$ for the 1-D system of isentropic gases. If the Mach number $M=\frac{1}{\sqrt{(K \gamma)}}$ is larger than $\frac{2}{\gamma-1}$ then an explicit solution in [19] proves that the solution develops a void region; if $0<M<\frac{2}{\gamma-1}$ then there is no void region but a region of small density. In Fig. 3 we illustrate numerically this fact from our method by solving the ODEs $(16,17,63,64)$ with the explicit Euler order one method. We present two tests; they are observed to be in agreement with the result in [19]. In both cases, $\gamma=1.5$. Close to the critical value $M=\frac{2}{\gamma-1}$, we have observed a very progressive transition so we have chosen two values of $M$ significantly different from the critical value. Denoting by $h$ the space step, $\Delta t$ the time step, $N$ the number of iterations and $\alpha$ an averaging parameter to model the convolution in (64) (by the replacement 

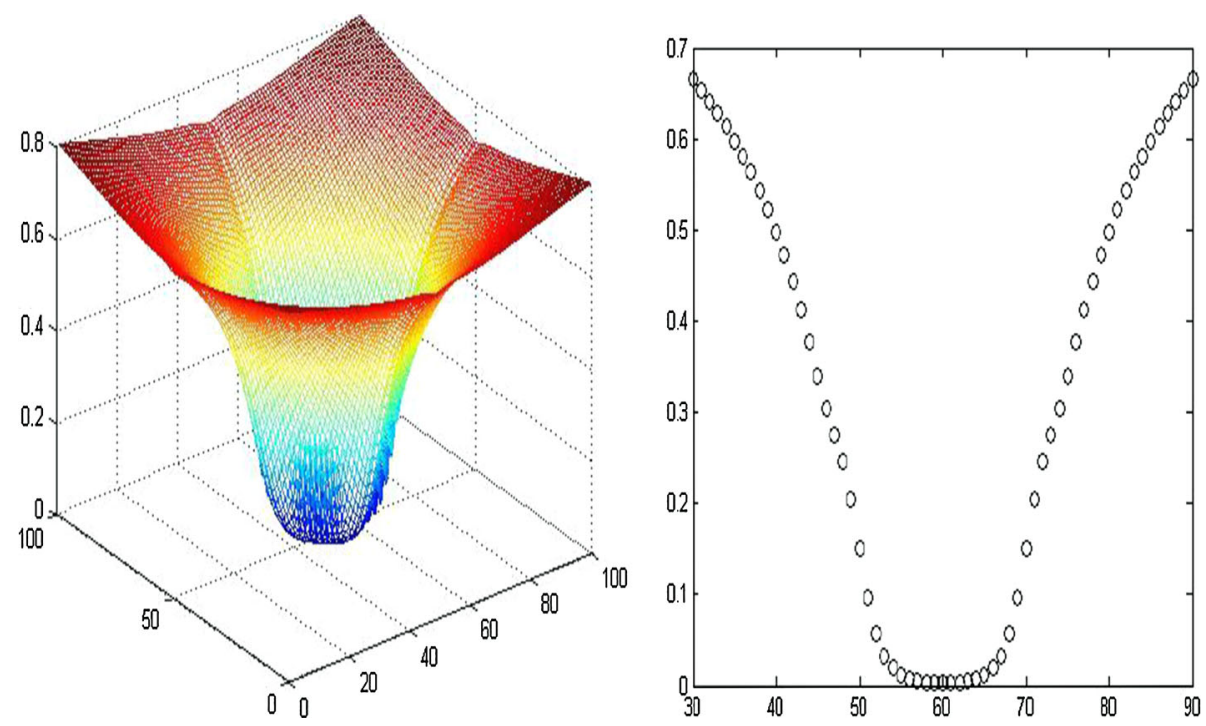

FIG. 4. A 2-D numerical solution in cartesian coordinates ignoring the rotational symmetry, with the explicit Euler order one scheme for the solution of the ODEs. One cannot say if the origin is a void region or a region with very small density. From the tests in [19] it is a void region

of $\Phi_{i}$ by $\tilde{\Phi}_{i}=\alpha \Phi_{i-1}+(1-2 \alpha) \Phi_{i}+\alpha \Phi_{i+1}$ and same replacement applied again to $\left.\partial_{x} \tilde{\Phi}_{i}=\frac{\tilde{\Phi}_{i+1}-\tilde{\Phi}_{i-1}}{2 h}\right)$, the chosen values of $(h, \Delta t, N, \alpha)$ are $(0.333,0.0167,90,0.3)$ for the left panel and $(0.333,0.0267,56,0.01)$ for the right panel.

To illustrate our method in 2-D, Fig. 4 produces a void region test: $\rho_{0}=1$, and radial velocity of modulus $=1$ directed toward the exterior. We apply the Euler order one scheme to the three ODEs ignoring on purpose the rotational symmetry. This is a case $\left(\gamma=1.5, M=\frac{5}{\gamma-1}\right)$ for which the authors of [19] have obtained from their 1-D test a void region. In our case, the result (which is a really $2-\mathrm{D}$ calculation) is too coarse to decide between a void region or a region with very small density. The values are $(h, \Delta t, n, \alpha)=(0.0556,0.0278,28,0.1)$, and according to the method in Sect. 2 , we have changed $u^{ \pm}, v^{ \pm}$into $u^{ \pm}+0.2, v^{ \pm}+0.2$ (vanishing viscosity).

\section{Sequence of approximate solutions to the shallow water equations}

In this section, we construct a weak asymptotic solution for the 2-D shallow water equations stated in the form

$$
\begin{aligned}
\partial_{t} h+\partial_{x}(h u)+\partial_{y}(h v) & =0, \\
\partial_{t}(h u)+\partial_{x}\left(h u^{2}\right)+\partial_{y}(h u v)+h \partial_{x} \Phi & =0, \\
\partial_{t}(h v)+\partial_{x}(h u v)+\partial_{y}\left(h v^{2}\right)+h \partial_{y} \Phi & =0, \\
\Phi & =g(h+a),
\end{aligned}
$$

where $h=h(x, y, t)$ is the water elevation, $(u, v)=(u(x, y, t), v(x, y, t))$ is the velocity vector in the $x, y$ directions, respectively, $a=a(x, y)$ is the bottom elevation assumed to be of class $\mathcal{C}^{1}$ and $g=9.8$. The initial condition and the function $a$ are assumed to be periodic in the $x, y$ variables (with period $2 \pi$ ). We give the proof in 1-D since the 2-D extension is straightforward as exposed in Sect. 2.

We state $h=\rho$ for convenience to benefit from the similarity with the other systems considered before. Then the 1-D shallow water equations are: 


$$
\begin{aligned}
\partial_{t} \rho+\partial_{x}(\rho u) & =0, \\
\partial_{t}(\rho u)+\partial_{x}\left(\rho u^{2}\right)+\rho \partial_{x} \Phi & =0, \\
\Phi & =g(\rho+a) .
\end{aligned}
$$

We approximate (69-71) by the following system of ODEs:

$$
\begin{gathered}
\frac{\mathrm{d}}{\mathrm{d} t} \rho(x, t, \epsilon)=\frac{1}{\epsilon}\left[\left(\rho u^{+}\right)(x-\epsilon, t, \epsilon)-(\rho|u|)(x, t, \epsilon)+\left(\rho u^{-}\right)(x+\epsilon, t, \epsilon)\right] \\
\frac{\mathrm{d}}{\mathrm{d} t}(\rho u)(x, t, \epsilon)=\frac{1}{\epsilon}\left[\left(\rho u u^{+}\right)(x-\epsilon, t, \epsilon)-(\rho u|u|)(x, t, \epsilon)+\left(\rho u u^{-}\right)(x+\epsilon, t, \epsilon)\right]-\rho(x, t, \epsilon) \partial_{x} \Phi(x, t, \epsilon), \\
\Phi(x, t, \epsilon)=g\left(\rho(., t, \epsilon) * \phi_{\epsilon^{\alpha}}\right)(x)+g a(x), \\
u(x, t, \epsilon)=\frac{(\rho u)(x, t, \epsilon)}{\rho(x, t, \epsilon)}
\end{gathered}
$$

for which we will prove that $\rho(x, t, \epsilon)>0$, thus permitting division.

We assume $\rho_{0}$ and $u_{0}$ are given with the properties $\rho_{0} \in L^{1}(\mathbb{T})$ and $u_{0} \in L^{\infty}(\mathbb{T})$ and that $\rho_{0}^{\epsilon}$ and $u_{0}^{\epsilon}$ are continuous regularizations of $\rho_{0}$ and $u_{0}$, respectively, with respective $L^{1}$ and $L^{\infty}$ bounds independent on $\epsilon$, and $\rho_{0}^{\epsilon}(x)>0 \forall x \in \mathbb{T}$, and satisfy $(20,21)$ at time $t=0$.

We first establish a priori inequalities to prove existence of a global solution to (72-75). For fixed $\epsilon>0$, we assume $(19,20,21)$. Then one obtains the a priori inequalities:

the statements (22 without the $\epsilon^{\beta}$ term), (23, 24, 25 with $2 \alpha$ in place of $3 \alpha$ ) and (26) hold as in Proposition 1.

The proof of the analog of (22) is identical to the proof of (22) in Proposition 1. For the proof of the analog of (23)

$$
\partial_{x} \Phi(x, t, \epsilon)=g\left[\rho(., t, \epsilon) *\left(\phi_{\epsilon^{\alpha}}\right)^{\prime}\right](x)+g a^{\prime}(x)
$$

i.e.,

$$
\partial_{x} \Phi(x, t, \epsilon)=g \int_{\mathbb{R}} \rho(x-y, t, \epsilon) \frac{1}{\epsilon^{2 \alpha}} \phi^{\prime}\left(\frac{y}{\epsilon^{\alpha}}\right) \mathrm{d} y+g a^{\prime}(x) .
$$

Therefore

$$
\left|\partial_{x} \Phi(x, t, \epsilon)\right| \leq \frac{\text { const }}{\epsilon^{2 \alpha}}\|\rho(., t, \epsilon)\|_{L^{1}(x-\operatorname{supp}(\phi))}+\text { const. }
$$

The proofs of the analogs of $(24,26)$ are similar to those in Proposition 1.

The existence of a global solution for fixed $\epsilon$ is obtained from these a priori estimates as in Sect. 2 .

It remains to prove that the solution of the system of ODEs (72-75) provides a weak asymptotic solution for system (69-71) when $\epsilon \rightarrow 0$. For $(69,70)$ the proof in Sect. 2 applies. For the state law $(71)$, we have to check that

$$
\int_{\mathbb{R}} \Phi(x, t, \epsilon) \psi(x) \mathrm{d} x=g \int_{\mathbb{R}} \rho(x, t, \epsilon) \psi(x) \mathrm{d} x+g \int_{\mathbb{R}} a(x) \psi(x) \mathrm{d} x+f(\epsilon),
$$

where $\Phi$ is given by (74) and where $f(\epsilon) \rightarrow 0$ when $\epsilon \rightarrow 0$. To check (76), it suffices from (74) to consider the integral

$$
\begin{aligned}
\int_{\mathbb{R}}\left[\rho(., t, \epsilon) * \phi_{\epsilon^{\alpha}}\right](x) \psi(x) \mathrm{d} x & =\int_{\mathbb{R}} \rho(x-y, t, \epsilon) \frac{1}{\epsilon^{\alpha}} \phi\left(\frac{y}{\epsilon^{\alpha}}\right) \psi(x) \mathrm{d} y \mathrm{~d} x=\int_{\mathbb{R}} \rho(x, t, \epsilon) \phi(\mu) \\
\psi\left(x+\epsilon^{\alpha} \mu\right) \mathrm{d} \mu \mathrm{d} x & =\int_{\mathbb{R}} \rho(x, t, \epsilon) \psi(x) \mathrm{d} x+\int_{\mathbb{R}} \rho(x, t, \epsilon) \phi(\mu)\left[\psi\left(x+\epsilon^{\alpha} \mu\right)-\psi(x)\right] d \mu \mathrm{d} x .
\end{aligned}
$$



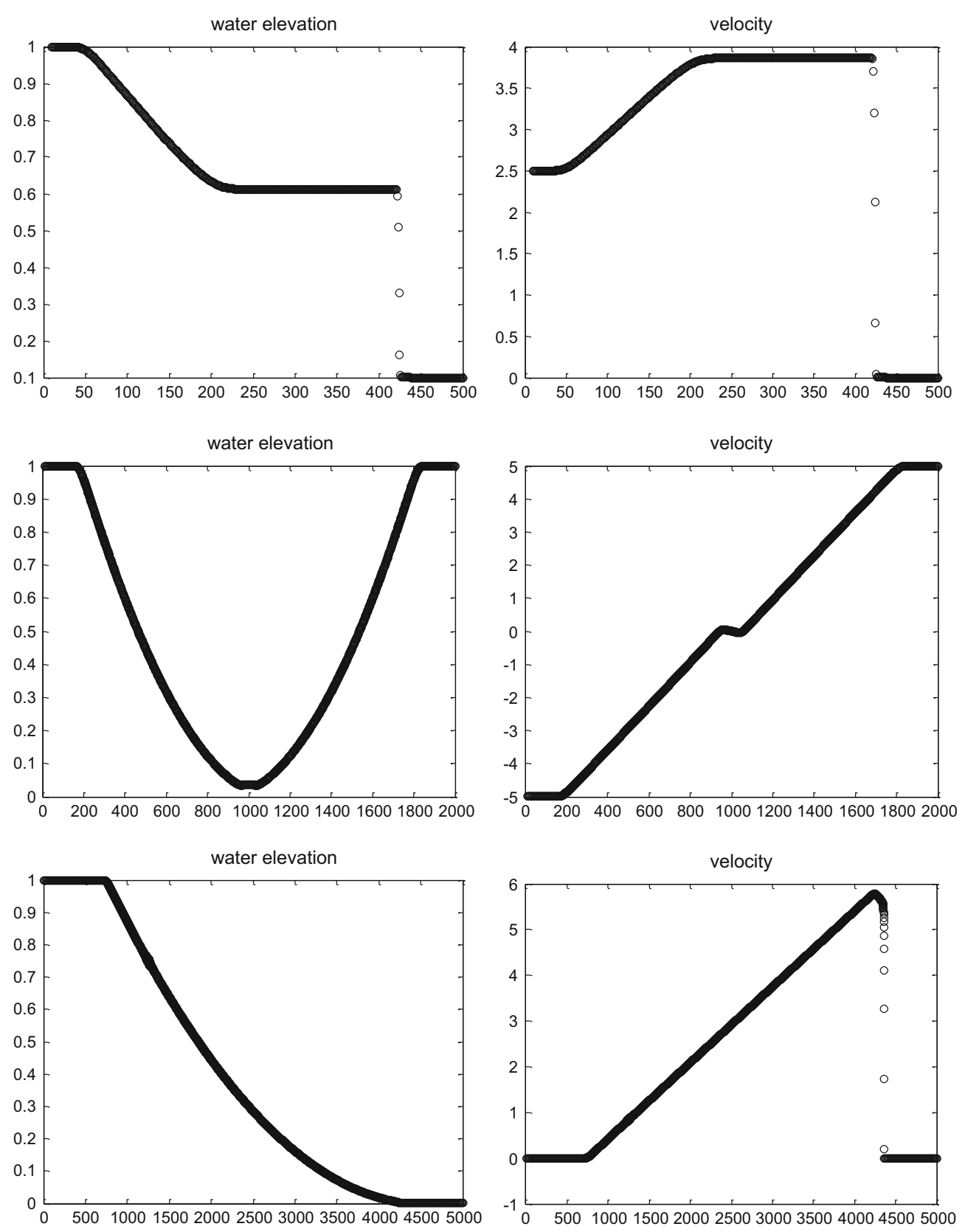

Fig. 5. From top to bottom the 1-D Toro tests 1, 2 and 3 on the equations shallow water in [35] pp. 109-124; left water elevation, right water velocity. The results were obtained with the explicit Euler order one method applied to the ODEs $(80,82)$. One observes coincidence with the exact solutions given in [35], although these tests have been chosen on purpose to be very demanding from the numerical viewpoint. One observes shocks and void regions in the top and bottom tests

The last term is $O\left(\epsilon^{\alpha}\right)$ from the $L_{l o c}^{1}$ property (22) of $\rho$ since integration takes place on a compact set. The 2-D proof is a direct adaptation of the 1-D proof as in Sect. 2.

Finally, we have proved: Let the initial conditions $\rho_{0} \in L^{1}(\mathbb{T}), u^{0} \in L^{\infty}(\mathbb{T}), \rho_{0} \geq 0$, $a$ a function of class $\mathcal{C}^{1}$ on $\mathbb{T}$. Approximate the initial conditions by a family $\left(\rho_{0}^{\epsilon}, u_{0}^{\epsilon}\right) \in(\mathcal{C}(\mathbb{T}))^{2}$, such that $\rho_{0}^{\epsilon}(x)>$ $0 \forall x,\left\|\rho_{0}-\rho_{0}^{\epsilon}\right\|_{L^{1}(\mathbb{T})} \rightarrow 0,\left|\left\|u_{0}\right\|_{\infty}-\left\|u_{0}^{\epsilon}\right\|_{\infty}\right| \rightarrow 0$ when $\epsilon \rightarrow 0$, then 

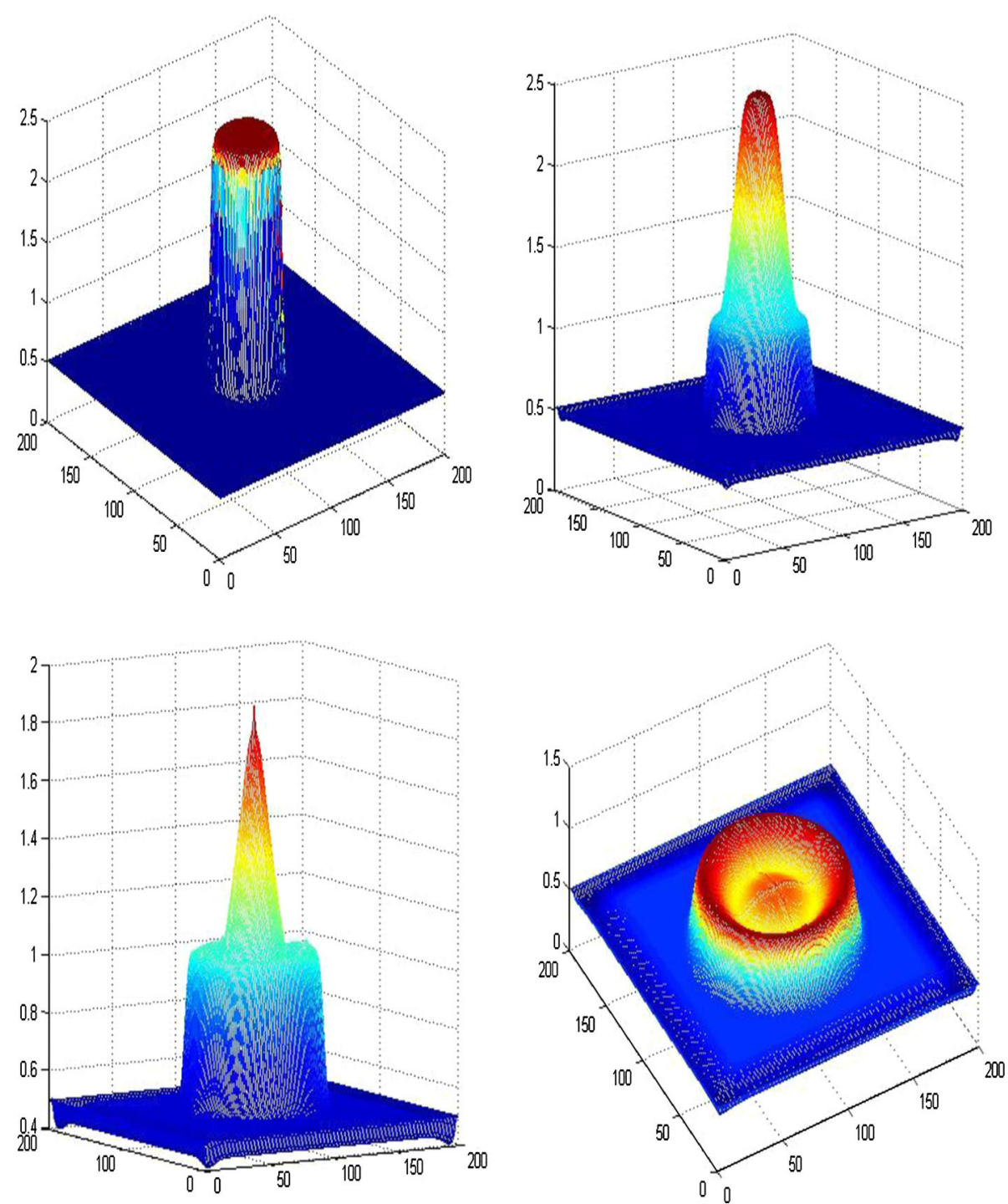

Fig. 6. From top left to bottom right the 2-D cylindrical dam break problem of Toro [35] at times $t=0,5,10,14$. One observes the correct results given in [35] although this test is demanding and one uses only the explicit Euler order one method for the ODEs in cartesian coordinates ignoring the rotational symmetry

Theorem 3. The solution of the system of ODEs (72-75), $0<\alpha<\frac{1}{4}$, with initial conditions $\left(\rho_{0}^{\epsilon}, u_{0}^{\epsilon}\right)$ provides a weak asymptotic solution (1) for the 1-D shallow water equations (69-71), which is global in time $t \in[0,+\infty[$ and in space $x \in \mathbb{T}$. The result holds as well in 2-D if $\alpha>0$ is small enough.

One obtains coherence with the classical analytic solution as in Proposition 2. Numerical tests from [35] have given results similar to those in Sects. 2 and 3, with the possible use of $v(u)$ in place of $u$. In Fig. 5, we have tested the three Riemann problem tests 1, 2 and 3 (from top to bottom) in [35] pp. 109-124. Top: We obtain the exact solution in test 1 with 500 space steps, $\mathrm{d} t=0.0001$, an averaging on 3 cells to represent the convolution (74) on the state law with coefficients $\alpha, 1-2 \alpha, \alpha$ with $\alpha=0.1$, no averaging needed on the initial condition and $\nu=0$ in the formula $v(u)=\left(u^{2}+\nu^{2}\right)^{\frac{1}{2}}$. Middle: We obtain 
the exact solution in test 2 with 2000 space steps, $\mathrm{d} t=0.00004$, an averaging for the state law (74) with $\alpha=0.1$, a similar averaging with $\alpha=0.1$ for the initial condition and $\nu=1$. Bottom: We obtain the exact solution in test 3 with 5000 space steps, $\mathrm{d} t=8.10^{-6}$, averagings with $\alpha=0.1$ for the state law (74) and the initial condition, $\nu=0.5$. Due to a void region in initial condition $(\rho=1$ on the left and 0 on the right), one has replaced the right value $\rho=0$ by $10^{-10}$ since the proof of weak asymptotic solution requests $\rho_{0}^{\epsilon}(x)>0 \forall x$. The initial data are apparent on both sides of the figures. The original tests [35] are not periodic, but one can imagine periods longer than the intervals under consideration to have a periodic problem on the time interval under consideration.

In Fig. 6 the initial condition (top left panel) is a cylinder of water of height $=2$ located on a flat surface of water of height $=0.5$. One observes the collapse under gravity of the column of water; $h=$ $0.005, r=\frac{\Delta t}{h}=0.1$, the averaging in $\Phi$ is obtained with $\alpha=0.1$; one transforms $u^{ \pm}, v^{ \pm}$into $u^{ \pm}+2, v^{ \pm}+2$ as in Sect. 2.

\section{Conclusion}

In view of providing a substitute of solutions that could provide a mathematical explanation of the results usually observed in scientific computing such as those exposed in $[25,26]$, we have constructed approximate solutions (up to any given accuracy in the sense of distributions) to the general Cauchy problem on the $n$-dimensional torus $\mathbb{T}^{n}, n=1,2,3$, for some standard equations of fluid dynamics in the presence of shocks and void regions.

Up to our knowledge, sequences of global approximate solutions to the general initial value problem for the equations of fluid dynamics with a complete mathematical proof that these approximate solutions tend to satisfy the equations had not been constructed previously. The statement of the method is based on the replacement of each equation by a nonlinear ordinary differential equation in a classical Banach space of functions. A priori estimates permit to prove existence-uniqueness of global solutions (in space and in positive time) for these ODEs. The continuity equation (mass conservation) gives a $L^{1}$ control on density which, from its use inside the state law giving pressure, permits from the Euler equations some control on velocity.

The method of proof allows numerical calculations from standard convergent numerical methods for ODEs such as the explicit Euler order 1 method and the RK4 method. They have given the known solutions in all tests.

Uniqueness of an "admissible" class of such sequences of approximate solutions, containing those constructed in this paper ("existence part"), such that all sequences in this class give same values for all time at the limit $\epsilon \rightarrow 0$ ("uniqueness part"), has been obtained so far only for scalar equations [13] where it has been proved that the method in this paper gives the Krushkov entropy solution at the limit $\epsilon \rightarrow 0$. Passage to the limit by some kind of weak compactness has not been considered so far.

\section{Acknowledgements}

The Author is very much indebted to the two referees for improvements of the original manuscript.

Open Access. This article is distributed under the terms of the Creative Commons Attribution 4.0 International License (http://creativecommons.org/licenses/by/4.0/), which permits unrestricted use, distribution, and reproduction in any medium, provided you give appropriate credit to the original author(s) and the source, provide a link to the Creative Commons license, and indicate if changes were made. 


\section{References}

1. Albeverio, S., Danilov, V.G.: Construction of global in time solutions to Kolmogorov-Feller pseudodifferential equations with a small parameter using characteristics. Mathematische Nachrichten 285, 426-439 (2012)

2. Albeverio, S., Rozanova, O.S., Shelkovich, V.M.: Transport and concentration processes in the multidimensional zero-pressure gas dynamics model with the energy conservation law. ArXiv:1101.581v1 (2011)

3. Albeverio, S., Shelkovich, V.M. : On delta shock problem. In: Rozanova, O. (ed.) Analytical Approaches to Multidimensional Balance Laws, chap. 2., pp. 45-88. Nova Science Publishers, New York (2005)

4. Choudhury, A.P., Joseph, K.T., Sahoo Manas, R.: Spherically symmetric solutions of multi-dimensional zero pressure gas dynamics system. J. Hyperbolic Diff. Eq. 11(2), 269-293 (2014)

5. Bouchut, F., Jin, S., Li, X.: Numerical approximation of pressureless and isothermal gas dynamics. SIAM J. Numer. Anal. 41(1), 135-158 (2003)

6. Chertock, A., Kurganov, A., Rykov, Y.: A new sticky particle method for pressure less gas dynamics. SIAM J. Numer. Anal. 45, 2408-2441 (2007)

7. Colombeau, M.: A method of projection of delta waves in a Godunov scheme and application to pressureless fluid dynamics. SIAM J. Numer. Anal. 48(5), 1900-1919 (2010)

8. Colombeau, M.: A consistent numerical scheme for self-gravitating fluid dynamics. Num. Methods PDEs 29(1), 79101 (2013)

9. Colombeau, M.: A simple numerical scheme for the 3-D system of ideal gases and a study of approximation in the sense of distributions. J. Comput. Appl. Math. 248, 15-30 (2013)

10. Colombeau, M.: Irregular shock wave solutions as continuations of the analytic solutions. Appl. Anal. doi:10.1080/ 00036811.2014.952290. ArXiv:1401.0285 (2014)

11. Colombeau, M.: Weak asymptotic methods for 3-D self-gravitating pressureless fluids; application to the creation and evolution of solar systems from the fully nonlinear Euler-Poisson equations. J. Math. Phys. 56, 061506 (2015)

12. Colombeau, M.: Asymptotic study of the initial value problem to a standard one pressure model of multifluid flows in nondivergence form. Preprint. ArXiv:1406.0122 (2014)

13. Work in redaction with E. Panov on scalar conservation laws.

14. de Costa Campos, L.M.B.: Complex Analysis with Applications to Flows and Fields. Maths and Physics for Science and Technology. Taylor and Francis, London (2011)

15. Danilov, V.G., Omel'yanov, G.A., Shelkovich, V.M.: Weak Asymptotic Method and Interaction of Nonlinear Waves. AMS Transl. 208, 33-164 (2003)

16. Danilov, V.G., Mitrovic, D.: Delta shock wave formation in the case of triangular hyperbolic system of conservation laws. J. Differ. Eq. 245, 3704-3734 (2008)

17. Danilov, V.G., Shelkovich, V.M.: Dynamics of propagation and interaction of $\delta$ shock waves in conservation law systems. J. Differ. Eq. 211, 333-381 (2005)

18. Danilov, V.G., Shelkovich, V.M.: Delta-shock wave type solution of hyperbolic systems of conservation laws. Q. Appl. Math. 63, 401-427 (2005)

19. Devault, K.J., Gremaud, P.A., Jenssen, H.K.: Numerical investigation of cavitation in multidimensional compressible flows. Siam J. Appl. Math. 67(6), 1675-1692

20. Evje, S., Flatten, T.: Hybrid flux-splitting Schemes for a common two fluid model. J. Comput. Phys. 192, 175$210(2003)$

21. Guyon, E., Hulin, J.P., Petit, L., Mitrescu, C.D.: Physical Hydrodynamics. Oxford University Press, Oxford (2001)

22. Joseph, K.T., Sahoo, M.R.: Boundary Riemann problems for the one dimensional adhesion model. Can. Appl. Math. Q. 19, 19-41 (2011)

23. Kmit, I., Kunzinger, M., Steinbauer, R.: Generalized solutions of the Vlasov-Poisson system with singular data. J. Math. Anal. Appl. 340(1), 575-587 (2008)

24. Kunzinger, M., Rein, G., Steinbauer, R., Teschl, G.: Global weak solution of the relativistic Vlasov-Klein Gordon system. Comm. Math. Phys. 238(1-2), 367-378 (2003)

25. Lax, P.D.: Mathematics and physics. Bull. AMS 45(1), 135-152 (2008)

26. Lax, P.D.: Computational fluid dynamics. J. Sci. Comput. 31(1-2), 185-193 (2007)

27. LeVeque, R.J.: The dynamics of pressureless dust clouds and delta waves. J. Hyperbolic Differ. Eq. 1, 315-327 (2004)

28. Nishida T. (1977) A note on a theorem of Nirenberg. J. Diff. Geom. 12, 629-633

29. Novotny, A., Straskraba, I.: Introduction to the mathematical theory of compressible flows. In: Oxford Lecture Series in Maths and its Applications (2008)

30. Panov, E.Yu., Shelkovich, V.M.: $\delta$ '-shock waves as a new type of solutions to systems of conservation laws. J. Differ. Eq. 228, 49-86 (2006)

31. Serre, D.: Von Neumann's comments about existence and uniqueness for the initial-boundary value problem in gas dynamics. Bull. AMS 47(1), 139-144 (2010) 
32. Shelkovich, V.M.: $\delta$ - and $\delta^{\prime}$-shock wave types of singular solutions of systems of conservation laws and transport and concentration processes. Russ. Math. Surv. 63(3), 405-601 (2008)

33. Shelkovich, V.M.: The Riemann problem admitting $\delta$-, $\delta$ '-shocks and vacuum states; the vanishing viscosity approach. J. Diff. Eq. 231, 459-500 (2006)

34. Sod, G.A.: A survey of several finite difference methods for systems of nonlinear hyperbolic conservation laws. J. Comput. Phys. 27, 1-31 (1978)

35. Toro, E.: Shock Capturing Methods for Free Surface Flows. Second edition. Wiley, New York (2001)

36. Toro, E.: Riemann Solvers and Numerical Methods for Fluid Dynamics. Springer, Berlin (1999)

37. Treves, F.: Basic Linear Partial Differential Equations. Academic Press, New York (1975)

38. Woodward, P., Colella, Ph.: The numerical simulation of two dimensional flows with strong shocks. J. Comput. Phys. 54, 115-173 (1984)

M. Colombeau

Instituto de Matemática e Estatística

Universidade de São Paulo

São Paulo

Brazil

e-mail: mcolombeau@ime.usp.br

(Received: November 19, 2014; revised: March 30, 2015) 